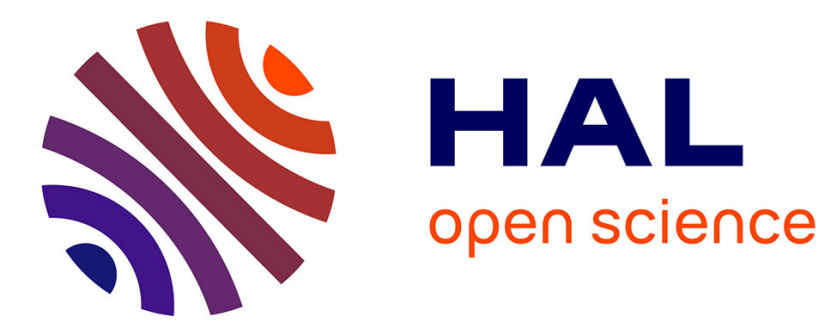

\title{
Finding scattering data for a time-harmonic wave equation with first order perturbation from the Dirichlet-to-Neumann map
}

Alexey Agaltsov

\section{To cite this version:}

Alexey Agaltsov. Finding scattering data for a time-harmonic wave equation with first order perturbation from the Dirichlet-to-Neumann map. 2015. hal-01110271

\section{HAL Id: hal-01110271 \\ https://hal.science/hal-01110271}

Preprint submitted on 27 Jan 2015

HAL is a multi-disciplinary open access archive for the deposit and dissemination of scientific research documents, whether they are published or not. The documents may come from teaching and research institutions in France or abroad, or from public or private research centers.
L'archive ouverte pluridisciplinaire HAL, est destinée au dépôt et à la diffusion de documents scientifiques de niveau recherche, publiés ou non, émanant des établissements d'enseignement et de recherche français ou étrangers, des laboratoires publics ou privés. 


\title{
Finding scattering data for a time-harmonic wave equation with first order perturbation from the Dirichlet-to-Neumann map
}

\author{
A. D. Agaltso $\chi^{1} 2$
}

We present formulas and equations for finding scattering data from the Dirichlet-to-Neumann map for a time-harmonic wave equation with first order perturbation with compactly supported coefficients. We assume that the coefficients are matrix-valued in general. To our knowledge, these results are new even for the general scalar case.

Keywords: inverse boundary value problems, inverse scattering, time-harmonic wave equation, Yang-Mills potentials

Subjects: partial differential equations, mathematical physics

AMS classification: 35R30 (Inverse problems), 35Q35 (PDEs in connection with fluid mechanics), 35Q40 (PDEs in connection with quantum mechanics)

\section{Introduction}

We consider the following equation:

$$
L \psi \stackrel{\text { def }}{=}-\Delta \psi-2 i \sum_{j=1}^{d} A_{j}(x) \partial_{x_{j}} \psi+V(x) \psi=E \psi, \quad x \in D \subset \mathbb{R}^{d},
$$

where $x=\left(x_{1}, \ldots, x_{d}\right), \partial_{x_{j}}=\partial / \partial x_{j}, \Delta=\partial_{x_{1}}^{2}+\cdots+\partial_{x_{d}}^{2}, E \in \mathbb{C}, d=2,3$,

$$
D \text { is a bounded open domain in } \mathbb{R}^{d} \text { with } \partial D \in C^{2} \text {, }
$$

$A_{1}, \ldots, A_{d}, V$ are sufficiently regular $M_{n}(\mathbb{C})$-valued functions on $\bar{D}$ and $M_{n}(\mathbb{C})$ is the set of $n \times n$ complex matrices.

We also assume that

$$
E \text { is not a Dirichlet eigenvalue for operator } L \text { in } D \text {. }
$$

\footnotetext{
${ }^{1}$ Centre de Mathématiques Appliquées, Ecole Polytechnique Route de Saclay 
Note that equation 1.1 can be written in the form

$$
\sum_{j=1}^{d}\left(-i \partial_{x_{j}}+A_{j}(x)\right)^{2} \psi+v(x) \psi=E \psi
$$

where

$$
v(x)=V(x)-\sum_{j=1}^{d} A_{j}^{2}(x)+i \sum_{j=1}^{d} \partial_{x_{j}} A_{j}(x) .
$$

For equation (1.1) (or 1.4) we consider the maps $\Phi(E), \Lambda(E)$ such that

$$
\begin{gathered}
\Phi(E)\left(\left.\psi\right|_{\partial D}\right)=\left.\frac{\partial \psi}{\partial \nu}\right|_{\partial D}, \\
\Lambda(E)\left(\left.\psi\right|_{\partial D}\right)=\left.\left(\frac{\partial \psi}{\partial \nu}+i \sum_{j=1}^{d} \nu_{j} A_{j} \psi\right)\right|_{\partial D},
\end{gathered}
$$

for all sufficiently regular solutions $\psi$ of 1.1 in $\bar{D}=D \cup \partial D$, for example for all $\psi \in C^{1}\left(\bar{D}, M_{n}(\mathbb{C})\right) \cap C^{2}\left(D, M_{n}(\mathbb{C})\right)$ satisfying $(1.1)$, where $\nu=\left(\nu_{1}, \ldots, \nu_{d}\right)$ is the unit exterior normal to $\partial D$. The map $\Phi(E)$ is known as the Dirichlet-toNeumann map for equation (1.1) in $D$.

In a similar way with [IN1], assumption (1.3) can be dropped by considering an appropriate Robin-to-Robin map instead of the Dirichlet-to-Neumann map.

Note that $\Lambda(E)$ is invariant with respect to the gauge transformations

$$
\begin{aligned}
A_{j} & \rightarrow g A_{j} g^{-1}+i\left(\partial_{x_{j}} g\right) g^{-1}, \quad j=1, \ldots, d, \\
v & \rightarrow g v g^{-1},
\end{aligned}
$$

where $g$ is a sufficiently regular $M_{n}(\mathbb{C})$-valued function on $\bar{D}$ with $\operatorname{det} g(x) \neq 0$, $x \in \bar{D}$ and $g(x)=\operatorname{Id}_{n}$ on $\partial D$, where $\operatorname{Id}_{n}$ is the identity $n \times n$ matrix. Note also that $\Phi(E)$ is invariant with respect to the gauge transformations (1.6) under the additional assumption that $\sum_{j=1}^{d} \nu_{j} \partial_{x_{j}} g=0$ on $\partial D$. Furthermore, if $\sum_{j=1}^{d} \nu_{j} A_{j}=0$ on $\partial D$ (in particular, if $A_{1}, \ldots, A_{d}$ have compact supports in $D)$, then $\Lambda(E)=\Phi(E)$. Besides, if $A_{1}, \ldots, A_{d}$ are known on $\partial D$, one can easily compute $\Lambda(E)$ given $\Phi(E)$ and vice versa.

For $n=1$ equation (1.4) can be considered as a Schrödinger equation at fixed energy $E$ with magnetic potential $A=\left(A_{1}, \ldots, A_{d}\right)$ and electric potential $v$, see, e.g., Refs. [HN1], [ER1], [ER3].

Equation (1.4) for $n \geq 2$ with Hermitian matrices $A_{1}, \ldots, A_{d}$ and with scalar matrix $v$ can be considered as a Schrödinger equation for a particle in an external Yang-Mills field, see Refs. [ST1], [ST2], [TU], [ER2].

Besides, equation (1.1) for $n=1$ is a model equation for the time-harmonic $\left(e^{-i \omega t}\right)$ acoustic pressure $\psi$ in a moving fluid, see, e.g., Refs. [RW], [RE]. In this setting

$$
E=\left(\frac{\omega}{c_{0}}\right)^{2}, \quad A_{j}(x)=\frac{\omega}{c_{0}} u_{j}(x), \quad V(x)=\left(1-n^{2}(x)\right)\left(\frac{\omega}{c_{0}}\right)^{2},
$$


where $j=1, \ldots, d, c_{0}$ is a reference sound speed, $n$ is a scalar index of refraction, $u=\left(u_{1}, \ldots, u_{d}\right)$ is a normalized fluid velocity vector.

In addition, for $n \geq 2, d=2$, equation (1.1) arises as a wave equation in the mode representation for a time-harmonic acoustic pressure $\psi$ in a moving fluid in a three-dimensional cylindrical domain of finite height and with base $D$, see Ref. [BBS].

We also consider equation (1.1) in the entire space:

$$
L \psi \equiv-\Delta \psi-2 i \sum_{j=1}^{d} A_{j}(x) \partial_{x_{j}} \psi+V(x) \psi=E \psi, \quad x \in \mathbb{R}^{d},
$$

where $A_{1}, \ldots, A_{d}, V$ are sufficiently regular $M_{n}(\mathbb{C})$-valued functions with sufficient decay at infinity.

There are scattering functions $\psi^{+}, f$ and Faddeev-type generalized scattering functions $\psi, h$ and $\psi_{\gamma}, h_{\gamma}$ associated with equation (1.7).

Functions $\psi^{+}, f$ can be defined as follows:

$$
\begin{gathered}
\psi^{+}(x, k)=e^{i k x} \operatorname{Id}_{n}+\int_{\mathbb{R}^{d}} G^{+}(x-y, k) \times \\
\times\left(-2 i \sum_{j=1}^{d} A_{j}(y) \partial_{y_{j}}+V(y)\right) \psi^{+}(y, k) d y \\
G^{+}(x, k)=-(2 \pi)^{-d} \int_{\mathbb{R}^{d}} \frac{e^{i \xi x} d \xi}{\xi^{2}-k^{2}-i 0},
\end{gathered}
$$

where $x \in \mathbb{R}^{d}, k \in \mathbb{R}^{d} \backslash 0$;

$$
f(k, l)=(2 \pi)^{-d} \int_{\mathbb{R}^{d}} e^{-i l x}\left(-2 i \sum_{j=1}^{d} A_{j}(x) \partial_{x_{j}}+V(x)\right) \psi^{+}(x, k) d x,
$$

where $k \in \mathbb{R}^{d} \backslash 0, l \in \mathbb{R}^{d}$. Actually, we consider 1.8 and its differentiated versions, where $\partial_{x_{j}}, j=1, \ldots, d$, are applied to both sides of (1.8), as a system of coupled linear integral equations for $\psi^{+}, \partial_{x_{j}} \psi^{+}, j=1, \ldots, d$.

Functions $\psi$ and $h$ are defined as follows:

$$
\begin{gathered}
\psi(x, k)=e^{i k x} \operatorname{Id}_{n}+\int_{\mathbb{R}^{d}} G(x-y, k) \times \\
\times\left(-2 i \sum_{j=1}^{d} A_{j}(y) \partial_{y_{j}}+V(y)\right) \psi(y, k) d y \\
G(x, k)=e^{i k x} g(x, k), \quad g(x, k)=-(2 \pi)^{-d} \int_{\mathbb{R}^{d}} \frac{e^{i \xi x} d \xi}{\xi^{2}+2 k \xi},
\end{gathered}
$$


where $x \in \mathbb{R}^{d}, k \in \mathbb{C}^{d} \backslash \mathbb{R}^{d}$;

$$
h(k, l)=(2 \pi)^{-d} \int_{\mathbb{R}^{d}} e^{-i l x}\left(-2 i \sum_{j=1}^{d} A_{j}(x) \partial_{x_{j}}+V(x)\right) \psi(x, k) d x,
$$

where $k, l \in \mathbb{C}^{d} \backslash \mathbb{R}^{d}, \operatorname{Im} k=\operatorname{Im} l, k^{2}=l^{2}$. In a similar way with (1.8), we consider (1.11) and its differentiated versions as a system of coupled linear integral equations for $\psi, \partial_{x_{j}} \psi$, or, more precisely, for $\mu, \partial_{x_{j}} \mu, j=1, \ldots, d$, where $\psi=e^{i k x} \mu$.

Finally, functions $\psi_{\gamma}$ and $h_{\gamma}$ are defined as follows:

$$
\psi_{\gamma}(x, k)=\psi(x, k+i 0 \gamma), \quad h_{\gamma}(k, l)=h(k+i 0 \gamma, l+i 0 \gamma),
$$

where $x \in \mathbb{R}^{d}, k, l \in \mathbb{R}^{d} \backslash 0, k^{2}=l^{2}, \gamma \in S^{d-1}$, and $S^{d-1}$ is the unit sphere in $\mathbb{R}^{d}$.

Note that the history of functions $\psi, h$ and $\psi_{\gamma}, h_{\gamma}$ goes back to [Fa1], [Fa2].

Functions $f(k, l)$ and $h_{\gamma}(k, l)$, where $k, l \in \mathbb{R}^{d} \backslash 0, k^{2}=l^{2}=E, \gamma \in S^{d-1}$, and $h(k, l)$, where $k, l \in \mathbb{C}^{d} \backslash \mathbb{R}^{d}, \operatorname{Im} k=\operatorname{Im} l, k^{2}=l^{2}=E$, are considered as the scattering data $S_{E}$ for equation (1.7) at fixed $E \in(0,+\infty)$. Function $h(k, l), k$, $l \in \mathbb{C}^{d} \backslash \mathbb{R}^{d}, \operatorname{Im} k=\operatorname{Im} l, k^{2}=l^{2}=E$, is considered as the scattering data $S_{E}$ for equation (1.7) at fixed $E \in \mathbb{C} \backslash(0,+\infty)$.

In a similar way with the map $\Lambda(E)$, scattering data $S_{E}$ is invariant with respect to gauge transformations $(1.6)$, where $g$ is a sufficiently regular $M_{n}(\mathbb{C})$ valued function on $\mathbb{R}^{d}$ decaying fast enough at infinity with $\operatorname{det} g(x) \neq 0$ for $x \in \mathbb{R}^{d}$, see, e.g., Ref. [AN] for the case $n=1$.

Let $D$ be a fixed domain satisfying $(1.2)$. Let

$$
A_{1}, \ldots, A_{d}, V \in C_{c}^{0, \alpha}\left(D, M_{n}(\mathbb{C})\right) \text { for some } 0<\alpha \leq 1,
$$

where $C_{c}^{0, \alpha}\left(D, M_{n}(\mathbb{C})\right)$ denotes the space of $M_{n}(\mathbb{C})$-valued component-wise Höldercontinuous functions with compact support in $D$. As it was noted above, in the case of coefficients satisfying 1.15 the maps $\Phi(E)$ and $\Lambda(E)$ are the same. For coefficients $A_{1}, \ldots, A_{d}, V$ satisfying 1.15 we consider the Dirichlet-toNeumann map $\Phi(E)$ for equation 11.1$)$ and the scattering data $S_{E}$ for equation (1.7). In the latter case we define coefficients $A_{1}, \ldots, A_{d}, V$ outside of $D$ by zero matrices.

Problem 1.1. Given $\Phi(E)$ at fixed $E$ (or for $E$ in some fixed set) find $A_{1}, \ldots$, $A_{d}, V$ of 1.1) (modulo gauge transformations (1.6)).

More precisely, we develop the approach of [No1] (where this approach was suggested for $\left.n=1, A_{1}, \ldots, A_{d} \equiv 0\right)$ and reduce Problem 1.1 to the following inverse scattering problem for equation (1.7):

Problem 1.2. Given $S_{E}$ at fixed $E$ (or for $E$ in some fixed set) find $A_{1}, \ldots$, $A_{d}, V$ of 1.7) (modulo gauge transformations (1.6)). 
Concerning the results given in literature on Problem 1.1 without the assumption that $A_{1} \equiv 0, \ldots, A_{d} \equiv 0$, see, e.g., Refs. [NSU], [Pa], [FKSU], [KLU], [IY], [KU] for $n=1$ and Ref. [Es] for $n \geq 1$. Besides, see Refs. [NS1], [NS2] for the case $d=2, A_{1} \equiv 0, A_{2} \equiv 0, n \geq 1$. Concerning the results for the case $n=1, A_{1} \equiv 0, \ldots, A_{d} \equiv 0$, see Refs. [No4], [Buk], [No5], [BSSR], [IN2], [Sa] and references therein.

Concerning the results given in literature on Problem 1.2 without the assumption $A_{1} \equiv 0, \ldots, A_{d} \equiv 0$, see, e.g., Refs. [Sh], [HN2], [No2] (p. 457), [ER1], [ER3], [Ar], [Ni], [PSU], [AN] for $n=1$ and Refs. [HN3], [ER2], [Es], [Xi] for $n \geq 1$. The case $A_{1} \equiv 0, \ldots, A_{d} \equiv 0, n \geq 1$, was considered, e.g., in Ref. [NS2]. Concerning the results for the case $n=1, A_{1} \equiv 0, \ldots, A_{d} \equiv 0$, see Ref. [No6] and references therein.

The main results of the present work consist of Theorems 2.1 and 2.2 of Section 2. In Theorem 2.1 we give, in particular, formulas and equations for finding $S_{E}$ from $\Phi(E)-\Phi^{0}(E)$, where $S_{E}$ and $\Phi(E)$ correspond to coefficients $A_{1}, \ldots, A_{d}, V$ and $\Phi^{0}(E)$ corresponds to zero coefficients $A_{1}^{0} \equiv 0, \ldots, A_{d}^{0} \equiv 0$, $V^{0} \equiv 0$. In Theorem 2.2 we give a result on the solvability of equations of Theorem 2.1 .

In fact, the formulas and equations of Theorem 2.1 are also valid if either $V^{0}(x)$ is a diagonal matrix for all $x \in D$ or $V^{0}$ is a product of a constant matrix by a scalar function, see Theorems 2.1] and 2.2] of Section 2. In this case, the potential $V^{0}$ is supposed to be known. This generalization to the case when $V^{0}(x)$ is diagonal for all $x \in D$ is useful, in particular, in the framework of Problem 1.1 for the case of mode wave equation, see, e.g., [BBS] and Subsection 3.1 of [NS2].

Thus, due to the results of Theorems 2.1, 2.2, 2.1] 2.2] we reduced Problem 1.1 to Problem 1.2. As regards to methods of solving Problem 1.2 we refer to [AN], [Ar], [ER1], [ER2], [ER3], [Es], [HN1], [HN2], [HN3], [Ni], [No2] (p. 457), [No6], [NS2], [PSU], [Sh], [Xi] and references therein.

For the case when $n=1, A_{1} \equiv 0, \ldots, A_{d} \equiv 0, A_{1}^{0} \equiv 0, \ldots, A_{d}^{0} \equiv 0, V^{0} \equiv 0$, Theorems 2.1] and 2.2 were obtained for the first time in [No1]. These theorems were generalized to the case when $n=1, A_{1} \equiv 0, \ldots, A_{d} \equiv 0, A_{1}^{0} \equiv 0, \ldots$, $A_{d}^{0} \equiv 0, V^{0} \not \equiv 0$, in [No4]. In [NS2] the authors give formulas and equations for the case when $d=2, n \geq 1, A_{1} \equiv 0, A_{2} \equiv 0, A_{1}^{0} \equiv 0, A_{2}^{0} \equiv 0, V^{0} \not \equiv 0$. In the present paper we generalize these results to the case when $n \geq 1, A_{1} \not \equiv 0, \ldots$, $A_{d} \not \equiv 0, A_{1}^{0} \equiv 0, \ldots, A_{d}^{0} \equiv 0, V^{0} \not \equiv 0$. To our knowledge, these results are new even for the general scalar case when $n=1$ and $V^{0} \equiv 0$.

The main results of the present work are presented in Section 2. 


\section{Main results}

Consider equation (1.7) under assumption 1.15). We define the sets $\mathcal{E}, \mathcal{E}_{\gamma}$, $\gamma \in S^{d-1}$, and $\mathcal{E}^{+}$, as follows:

$$
\begin{aligned}
& \mathcal{E}=\left\{\zeta \in \mathbb{C}^{d} \backslash \mathbb{R}^{d}: \text { equation } 1.11 \text { at } k=\zeta\right. \text { is not uniquely } \\
&\text { solvable for } \left.\psi=e^{i k x} \mu, \text { where } \mu \in W^{1, \infty}\left(\mathbb{R}^{d}, M_{n}(\mathbb{C})\right)\right\}, \\
& \mathcal{E}_{\gamma}=\left\{\zeta \in \mathbb{R}^{d} \backslash 0: \text { equation } 1.11 \text { at } k=\zeta+i 0 \gamma\right. \text { is not } \\
&\text { uniquely solvable for } \left.\psi \in W^{1, \infty}\left(\mathbb{R}^{d}, M_{n}(\mathbb{C})\right)\right\}, \\
& \mathcal{E}^{+}=\left\{\zeta \in \mathbb{R}^{d} \backslash 0 \text { : equation } 1.8 \text { at } k=\zeta+i 0 \zeta /|\zeta|\right. \text { is not } \\
&\text { uniquely solvable for } \left.\psi \in W^{1, \infty}\left(\mathbb{R}^{d}, M_{n}(\mathbb{C})\right)\right\} .
\end{aligned}
$$

The properties of sets $\mathcal{E}, \mathcal{E}_{\gamma}, \mathcal{E}^{+}$are similar to the properties of the analogs of sets $\mathcal{E}, \mathcal{E}_{\gamma}, \mathcal{E}^{+}$in the case when $n=1, A_{j} \equiv 0, j=1, \ldots, d$. For the properties of the latter sets see, e.g., Ref. [No4] and references therein. Restrictions in space and time prevent us from studying the properties of sets $\mathcal{E}, \mathcal{E}_{\gamma}, \mathcal{E}^{+}$in the present paper.

Theorem 2.1. Let $D$ satisfy 1.2 and $E$ be fixed. Suppose that $E$ is not a Dirichlet eigenvalue for operators $L$ and $-\Delta$ in $D$. Let $A_{1}, \ldots, A_{d}, V$ satisfy 1.15. Let $\Phi(E)$ correspond to coefficients $A_{1}, \ldots, A_{d}, V$ and $\Phi^{0}(E)$ correspond to coefficients $A_{1}^{0} \equiv 0, \ldots, A_{d}^{0} \equiv 0, V^{0} \equiv 0$. Denote by $\left(\Phi-\Phi^{0}\right)(x, y, E)$, $x, y \in \partial D$, the Schwartz kernel of operator $\Phi(E)-\Phi^{0}(E)$. Then the following formulas and equations hold:

$$
h(k, l)=(2 \pi)^{-d} \int_{\partial D} \int_{\partial D} e^{-i l x}\left(\Phi-\Phi^{0}\right)(x, y, E) \psi(y, k) d y d x,
$$

where $k, l \in \mathbb{C}^{d} \backslash \mathbb{R}^{d}, \operatorname{Im} k=\operatorname{Im} l, k^{2}=l^{2}=E, k \notin \mathcal{E}$;

$$
\begin{array}{r}
\psi(x, k)=e^{i k x} \operatorname{Id}_{n}+\int_{\partial D} A(x, y, k) \psi(y, k) d y, \quad x \in \partial D, \\
A(x, y, k)=\int_{\partial D} G(x-z, k)\left(\Phi-\Phi^{0}\right)(z, y, E) d z, \quad x, y \in \partial D,
\end{array}
$$

where $k \in \mathbb{C}^{d} \backslash\left(\mathbb{R}^{d} \cup \mathcal{E}\right), k^{2}=E$, and $G$ is defined in formula 1.12,

$$
h_{\gamma}(k, l)=(2 \pi)^{-d} \int_{\partial D} \int_{\partial D} e^{-i l x}\left(\Phi-\Phi^{0}\right)(x, y, E) \psi_{\gamma}(y, k) d y d x,
$$

where $\gamma \in S^{d-1}, k, l \in \mathbb{R}^{d} \backslash 0, k^{2}=l^{2}=E, k \notin \mathcal{E}_{\gamma}$,

$$
\begin{gathered}
\psi_{\gamma}(x, k)=e^{i k x} \operatorname{Id}_{n}+\int_{\partial D} A_{\gamma}(x, y, k) \psi_{\gamma}(y, k) d y, \quad x \in \partial D \\
A_{\gamma}(x, y, k)=\int_{\partial D} G_{\gamma}(x-z, k)\left(\Phi-\Phi^{0}\right)(z, y, E) d z, \quad x, y \in \partial D \\
G_{\gamma}(x, k) \stackrel{\text { def }}{=} G(x, k+i 0 \gamma), \quad x \in \mathbb{R}^{d},
\end{gathered}
$$


where $\gamma \in S^{d-1}, k \in \mathbb{R}^{d} \backslash\left(0 \cup \mathcal{E}_{\gamma}\right), k^{2}=E$;

$$
f(k, l)=(2 \pi)^{-d} \int_{\partial D} \int_{\partial D} e^{-i l x}\left(\Phi-\Phi^{0}\right)(x, y, E) \psi^{+}(y, k) d y d x,
$$

where $k, l \in \mathbb{R}^{d} \backslash 0, k^{2}=l^{2}=E, k \notin \mathcal{E}^{+}$,

$$
\begin{gathered}
\psi^{+}(x, k)=e^{i k x} \operatorname{Id}_{n}+\int_{\partial D} A^{+}(x, y, k) \psi^{+}(y, k) d y, \quad x \in \partial D, \\
A^{+}(x, y, k)=\int_{\partial D} G^{+}(x-z, k)\left(\Phi-\Phi^{0}\right)(z, y, E) d z, \quad x, y \in \partial D,
\end{gathered}
$$

where $k \in \mathbb{R}^{d} \backslash\left(0 \cup \mathcal{E}^{+}\right), k^{2}=E$, and $G^{+}$is defined in formula 1.9).

Actually, we consider 2.5, 2.8$), 2.12$ as integral equations for finding $\psi$, $\psi_{\gamma}, \psi^{+}$, respectively, from $\Phi(E)-\Phi^{0}(E)$.

In addition, we consider (2.4), 2.7), 2.11) as explicit formulas for finding $h, h_{\gamma}, f$ from $\Phi(E)-\Phi^{0}(E)$ and $\psi, \psi_{\gamma}, \psi^{+}$, respectively.

For fixed $0<\beta \leq 1$ we denote by $C^{1, \beta}\left(\partial D, M_{n}(\mathbb{C})\right)$ the Banach space of functions from $C^{1}\left(\partial \bar{D}, M_{n}(\mathbb{C})\right)$ with component-wise Hölder-continuous derivatives.

Theorem 2.2. Let the assumptions of Theorem 2.1 be fulfilled. Let $0<\beta<1$ be fixed.

1. Fix $k \in \mathbb{C}^{d} \backslash \mathbb{R}^{d}, k^{2}=E$. Then equation $(2.5)$ is a Fredholm integral equation of second kind for $\psi \in C^{1, \beta}\left(\partial D, M_{n}(\mathbb{C})\right)$ which is uniquely solvable if and only if $k \notin \mathcal{E}$.

2. Fix $\gamma \in S^{d-1}, k \in \mathbb{R}^{d} \backslash 0, k^{2}=E$. Then equation $(2.8)$ is a Fredholm integral equation of second kind for $\psi_{\gamma} \in C^{1, \beta}\left(\partial D, M_{n}(\mathbb{C})\right)$ which is uniquely solvable if and only if $k \notin \mathcal{E}_{\gamma}$.

3. Fix $k \in \mathbb{R}^{d} \backslash 0, k^{2}=E$. Then equation (2.12) is a Fredholm integral equation of second kind for $\psi^{+} \in C^{1, \beta}\left(\partial D, M_{n}(\mathbb{C})\right)$ which is uniquely solvable if and only if $k \notin \mathcal{E}^{+}$.

In fact, Theorems 2.1 and 2.2 are particular cases of more general Theorems 2.1 and 2.2 given below. To formulate these results we need to introduce some notations.

Let coefficients $A_{1}^{0}, \ldots, A_{d}^{0}, V^{0}$ on $\mathbb{R}^{d}$ satisfy

$$
A_{1}^{0} \equiv 0, \ldots, A_{d}^{0} \equiv 0,
$$

and either

$$
V^{0}(x) \text { be a diagonal matrix for all } x,
$$

or

$$
\begin{aligned}
& V^{0}=\mathcal{V}^{0} v^{0}, \text { where } \mathcal{V}^{0} \in M_{n}(\mathbb{C}) \\
& \text { and } v^{0} \text { is a slalar function of } x .
\end{aligned}
$$


We also suppose that $V^{0}$ is zero outide of $D$ and coefficients $A_{1}^{0}, \ldots, A_{d}^{0}, V^{0}$ restricted to $D$ satisfy (1.15).

Define $L_{V^{0}}, \mathcal{E}_{V^{0}}, \mathcal{E}_{V^{0}, \gamma}, \gamma \in S^{d-1}$, and $\mathcal{E}_{V^{0}}^{+}$by formulas 1.1 , (2.1), 2.2 (2.3), respectively, using coefficients $A_{1}^{0} \equiv 0, \ldots, A_{d}^{0} \equiv 0, V^{\sigma}$ in (1.1), (1.11), (1.8) instead of $A_{1}, \ldots, A_{d}, V$.

Note that, in fact, in the definition of set $\mathcal{E}_{V^{0}}$ (or sets $\mathcal{E}_{V^{0}, \gamma}, \mathcal{E}_{V^{0}}^{+}$) it is sufficient to consider the solvability of corresponding equations for $\psi=e^{i k x} \mu$ with $\mu \in L^{\infty}\left(\mathbb{R}^{d}, M_{n}(\mathbb{C})\right)$ (for $\psi \in L^{\infty}\left(\mathbb{R}^{d}, M_{n}(\mathbb{C})\right.$ ), respectively).

We consider the functions $R^{0}, R_{\gamma}^{0}, \gamma \in S^{d-1}$, and $R^{+, 0}$ defined as follows:

$$
R^{0}(x, y, k)=G(x-y, k) \operatorname{Id}_{n}+\int_{\mathbb{R}^{d}} G(x-z, k) V^{0}(z) R^{0}(z, y, k) d z,
$$

where $x, y \in \mathbb{R}^{d}, k \in \mathbb{C}^{d} \backslash \mathbb{R}^{d}$ and $G$ is defined in formula 1.12 ;

$$
\begin{gathered}
R_{\gamma}^{0}(x, y, k) \stackrel{\text { def }}{=} R^{0}(x, y, k+i 0 \gamma), \\
R^{+, 0}(x, y, k) \stackrel{\text { def }}{=} R_{k /|k|}^{0}(x, y, k),
\end{gathered}
$$

where $x, y \in \mathbb{R}^{d}, k \in \mathbb{R}^{d} \backslash 0, \gamma \in S^{d-1}$.

We consider 2.17) at fixed $y, k$ as an integral equation for

$$
R^{0}(x, y, k)=G(x-y, k) \operatorname{Id}_{n}+e^{i k(x-y)} r^{0}(x, y, k),
$$

where $r^{0}(\cdot, y, k) \in L^{\infty}\left(\mathbb{R}^{d}, M_{n}(\mathbb{C})\right)$.

It follows from 2.17, 2.20 that $r^{0}$ satisfies the following equation:

$$
\begin{gathered}
r^{0}(x, y, k)=\int_{\mathbb{R}^{d}} g(x-z, k) V^{0}(z) g(z-y, k) d z \\
+\int_{\mathbb{R}^{d}} g(x-z, k) V^{0}(z) r^{0}(z, y, k) d y,
\end{gathered}
$$

where $x, y \in \mathbb{R}^{d}$ and $g$ is defined in formula 1.12 .

Note that under assumption 1.15 for coefficients $A_{1}^{0} \equiv 0, \ldots, A_{d}^{0} \equiv 0, V^{0}$ the following statements are true:

1. Fix $k \in \mathbb{C}^{d} \backslash \mathbb{R}^{d}$. Then equation (2.21) is uniquely solvable for $r^{0}(\cdot, y, k) \in$ $L^{\infty}\left(\mathbb{R}^{d}, M_{n}(\mathbb{C})\right)$ for any $y \in \mathbb{R}^{d}$ if and only if $k \notin \mathcal{E}_{V^{0}}$.

2. Fix $\zeta \in \mathbb{R}^{d} \backslash 0, \gamma \in S^{d-1}$. Then equation (2.21) with $k=\zeta+i 0 \gamma$ is uniquely solvable for $r^{0}(\cdot, y, k) \in L^{\infty}\left(\mathbb{R}^{d}, M_{n}(\mathbb{C})\right)$ for any $y \in \mathbb{R}^{d}$ if and only if $\zeta \notin \mathcal{E}_{V^{0}, \gamma}$.

3. Fix $\zeta \in \mathbb{R}^{d} \backslash 0$. Then equation (2.21) with $k=\zeta+i 0 \zeta /|\zeta|$ is uniquely solvable for $r^{0}(\cdot, y, k) \in L^{\infty}\left(\mathbb{R}^{d}, M_{n}(\mathbb{C})\right)$ for any $y \in \mathbb{R}^{d}$ if and only if $\zeta \notin \mathcal{E}_{V^{0}}^{+}$. 
Besides, if equation (2.21) at fixed $k$ is uniquely solvable for $r^{0}(\cdot, y, k) \in$ $L^{\infty}\left(\mathbb{R}^{d}, M_{n}(\mathbb{C})\right)$ for any $y \in \mathbb{R}^{d}$, then function $r^{0}$ has the following properties:

$$
\begin{aligned}
& r^{0}(\cdot, \cdot, k) \in C\left(\mathbb{R}^{d} \times \mathbb{R}^{d}, M_{n}(\mathbb{C})\right) \cap L^{\infty}\left(\mathbb{R}^{d} \times \mathbb{R}^{d}, M_{n}(\mathbb{C})\right) \text { at fixed } k, \\
& \int_{\mathbb{R}^{d}} g(x-z, k) V^{0}(z) r^{0}(z, y, k) d z=\int_{\mathbb{R}^{d}} r^{0}(x, z, k) V^{0}(z) g(z-y, k) d z,
\end{aligned}
$$

where $x, y \in \mathbb{R}^{d}$.

We also consider the function $\widetilde{\psi}_{\gamma}^{0}$ defined as follows:

$$
\widetilde{\psi}_{\gamma}^{0}(x, k, l)=e^{i l x} \operatorname{Id}_{n}+\int_{\mathbb{R}^{d}} G_{\gamma}(x-y, k) V^{0}(y) \widetilde{\psi}_{\gamma}^{0}(y, k, l) d y,
$$

where $x \in \mathbb{R}^{d}, k, l \in \mathbb{R}^{d} \backslash 0, k^{2}=l^{2}, \gamma \in S^{d-1}$. We consider (2.24) at fixed $k, l$, $\gamma$ as an integral equation for $\widetilde{\psi}_{\gamma}^{0}(\cdot, k, l) \in L^{\infty}\left(\mathbb{R}^{d}, M_{n}(\mathbb{C})\right)$.

Theorem 2.1. Let $D$ satisfy $(1.2)$ and $E$ be fixed. Suppose that $E$ is not a Dirichlet eigenvalue for operators $L, L_{V^{0}}$ and $-\Delta$ in D. Consider two sets of coefficients $A_{1}, \ldots, A_{d}, V$ and $A_{1}^{0}, \ldots, A_{d}^{0}, V^{0}$, satisfying 1.15 . Let $A_{1}^{0}, \ldots$, $A_{d}^{0}, V^{0}$ satisfy (2.14) and either 2.15) or 2.16). Let $\Phi, \psi, h, \psi_{\gamma}, h_{\gamma}, \psi^{+}, f$, $\mathcal{E}, \mathcal{E}_{\gamma}, \mathcal{E}^{+}$correspond to $A_{1}, \ldots, A_{d}, V$ (as defined above) and $\Phi_{V^{0}}, \psi^{0}, h^{0}$, $\psi_{\gamma}^{0}, \widetilde{\psi}_{\gamma}^{0}, h_{\gamma}^{0}, \psi^{+, 0}, f^{0}, R^{0}, R_{\gamma}^{0}, R^{+, 0}, \mathcal{E}_{V^{0}}, \mathcal{E}_{V^{0}, \gamma}, \mathcal{E}_{V^{0}}^{+}$correspond to $A_{1}^{0}, \ldots, A_{d}^{0}$, $V^{0}$ (as defined above with coefficients $A_{1}^{0}, \ldots, A_{d}^{0}, V^{0}$ instead of $A_{1}, \ldots, A_{d}$, $V)$. Denote by $\left(\Phi-\Phi_{V^{0}}\right)(x, y, E), x, y \in \partial D$, the Schwartz kernel of operator $\Phi(E)-\Phi_{V^{0}}(E)$. Then the following formulas and equations hold:

$$
h(k, l)=h^{0}(k, l)+(2 \pi)^{-d} \int_{\partial D} \int_{\partial D} \psi^{0}(x,-l)\left(\Phi-\Phi_{V^{0}}\right)(x, y, E) \psi(y, k) d y d x,
$$

where $k, l \in \mathbb{C}^{d} \backslash \mathbb{R}^{d}, \operatorname{Im} k=\operatorname{Im} l, k^{2}=l^{2}=E, k \notin \mathcal{E} \cup \mathcal{E}_{V^{0}}$,

$$
\begin{array}{r}
\psi(x, k)=\psi^{0}(x, k)+\int_{\partial D} A(x, y, k) \psi(y, k) d y, \quad x \in \partial D, \\
A(x, y, k)=\int_{\partial D} R^{0}(x, z, k)\left(\Phi-\Phi_{V^{0}}\right)(z, y, E) d z, \quad x, y \in \partial D,
\end{array}
$$

where $k \in \mathbb{C}^{d} \backslash\left(\mathbb{R}^{d} \cup \mathcal{E} \cup \mathcal{E}_{V^{0}}\right), k^{2}=E$;

$$
\begin{gathered}
h_{\gamma}(k, l)=h_{\gamma}^{0}(k, l) \\
+(2 \pi)^{-d} \int_{\partial D} \int_{\partial D} \widetilde{\psi}_{-\gamma}^{0}(x,-k,-l)\left(\Phi-\Phi_{V^{0}}\right)(x, y, E) \psi_{\gamma}(y, k) d y d x,
\end{gathered}
$$

where $\gamma \in S^{d-1}, k, l \in \mathbb{R}^{d} \backslash 0, k^{2}=l^{2}=E, k \notin \mathcal{E}_{\gamma} \cup \mathcal{E}_{V^{0}, \gamma}$,

$$
\begin{gathered}
\psi_{\gamma}(x, k)=\psi_{\gamma}^{0}(x, k)+\int_{\partial D} A_{\gamma}(x, y, k) \psi_{\gamma}(y, k) d y, \quad x \in \partial D, \\
A_{\gamma}(x, y, k)=\int_{\partial D} R_{\gamma}^{0}(x, z, k)\left(\Phi-\Phi_{V^{0}}\right)(z, y, E) d z, \quad x, y \in \partial D,
\end{gathered}
$$


where $\gamma \in S^{d-1}, k \in \mathbb{R}^{d} \backslash\left(0 \cup \mathcal{E}_{\gamma} \cup \mathcal{E}_{V^{0}, \gamma}\right), k^{2}=E$;

$f(k, l)=f^{0}(k, l)+(2 \pi)^{-d} \int_{\partial D} \int_{\partial D} \psi^{+, 0}(x,-l)\left(\Phi-\Phi_{V^{0}}\right)(x, y, E) \psi^{+}(y, k) d y d x$,

where $k, l \in \mathbb{R}^{d} \backslash 0, k^{2}=l^{2}=E, k \notin \mathcal{E}^{+} \cup \mathcal{E}_{V^{0}}^{+}$,

$$
\begin{aligned}
\psi^{+}(x, k) & =\psi^{+, 0}(x, k)+\int_{\partial D} A^{+}(x, y, k) \psi^{+}(y, k) d y, \quad x \in \partial D, \\
A^{+}(x, y, k) & =\int_{\partial D} R^{+, 0}(x, z, k)\left(\Phi-\Phi_{V^{0}}\right)(z, y, E) d z, \quad x, y \in \partial D,
\end{aligned}
$$

where $k \in \mathbb{R}^{d} \backslash\left(0 \cup \mathcal{E}^{+} \cup \mathcal{E}_{V^{0}}^{+}\right), k^{2}=E$.

In a similar way with Theorem 2.1 we consider 2.26, $2.29,2.32$ as integral equations for finding $\psi, \psi_{\gamma}, \psi^{+}$from $\Phi(E)-\Phi_{V^{0}}(E)$ and $\psi^{0}, R^{0} ; \psi_{\gamma}^{0}$, $R_{\gamma}^{0} ; \psi^{+, 0}, R^{+, 0}$, respectively.

We also consider (2.25), 2.28, 2.31) as explicit formulas for finding $h, h_{\gamma}$, $f$ from $\Phi(E)-\Phi_{V^{0}}(E)$ and $h^{0}, \psi^{0}, \psi ; h_{\gamma}^{0}, \widetilde{\psi}_{-\gamma}^{0}, \psi_{\gamma} ; f^{0}, \psi^{+, 0}, \psi^{+}$, respectively.

Theorem 2.1] is proved in Section 3.

Theorem 2.2. Let the assumptions of Theorem 2.1] be fulfilled. Let $0<\beta<1$ be fixed.

1. Fix $k \in \mathbb{C}^{d} \backslash\left(\mathbb{R}^{d} \cup \mathcal{E}_{V^{0}}\right), k^{2}=E$. Then equation $(2.26)$ is a Fredholm integral equation of second kind for $\psi \in C^{1, \beta}\left(\partial D, M_{n}(\mathbb{C})\right)$ which is uniquely solvable if and only if $k \notin \mathcal{E}$.

2. Fix $\gamma \in S^{d-1}, k \in \mathbb{R}^{d} \backslash\left(0 \cup \mathcal{E}_{V^{0}, \gamma}\right), k^{2}=E$. Then equation 2.29$)$ is a Fredholm integral equation of second kind for $\psi_{\gamma} \in C^{1, \beta}\left(\partial D, M_{n}(\mathbb{C})\right)$ which is uniquely solvable if and only if $k \notin \mathcal{E}_{\gamma}$.

3. Fix $k \in \mathbb{R}^{d} \backslash\left(0 \cup \mathcal{E}_{V^{0}}^{+}\right), k^{2}=E$. Then equation 2.32 is a Fredholm integral equation of second kind for $\psi^{+} \in C^{1, \beta}\left(\partial D, M_{n}(\mathbb{C})\right)$ which is uniquely solvable if and only if $k \notin \mathcal{E}^{+}$.

Theorem 2.2] is proved in Section 4.

Remark 2.1. Note that the proofs of equations and formulas of Theorems 2.1 2.1 remain valid without the assumption that coefficients $A_{1}, \ldots, A_{d}, V, V^{0}$ have compact supports in $D$. The assumption that the coefficients have compact supports in $D$ was introduced in order to simplify the choice of functional spaces for solving equations $2.5,(2.8),(2.12), 2.26),(2.29),(2.32)$ and related proofs of Theorems 2.2, 2.2],

It is important to note that equation (1.11) and formula 1.13 give much more stable way to find functions $\psi^{0}, h^{0}$ from $A_{1}^{0}, \ldots, A_{d}^{0}, V^{0}$ than equation (2.5) and formula 2.4 if $|\operatorname{Im} k|$ is sufficiently large. 
On the other hand, it is known that the solution to equation 2.26 will be relatively stable if the norm of the integral operator involved in this equation is less then 1 . If at fixed $k$ coefficients $A_{1}, \ldots, A_{d}$ are sufficiently small whereas coefficient $V$ is not small but is sufficiently close to coefficient $V^{0}$, then the integral operator in equation 2.26 will have much smaller norm than the integral operator in equation 2.5 (e.g., as a norm of operator on $C^{1, \beta}\left(\partial D, M_{n}(\mathbb{C})\right)$, $0<\beta<1$ ). In particular, the norm will be less then 1 and we will be able to use the method of successive approximations to solve 2.26). Hence equation (2.26) and formula 2.25 will give much more stable way to find $\psi$ and $h$ than equation 2.5 and formula 2.4 , respectively. For more details, see pp. 262-263 of Ref. [No4] and Section 3.2 of [NS2] for related discussion.

\section{Proof of Theorem 2.1}

\subsection{Integral identity}

Note that we have the identity

$$
\begin{gathered}
\int_{\partial D} u^{0}(x)\left(\Phi(E)-\Phi_{V^{0}}(E)\right)\left(\left.u\right|_{\partial D}\right)(x) d x \\
=\int_{D} u^{0}(x)\left(-2 i \sum_{j=1}^{d} A_{j}(x) \partial_{x_{j}}+V(x)-V^{0}(x)\right) u(x) d x .
\end{gathered}
$$

for any sufficiently regular $M_{n}(\mathbb{C})$-valued functions $u, u^{0}$ on $\bar{D}$ (for example, for $\left.u, u^{0} \in C^{2}\left(D, M_{n}(\mathbb{C})\right) \cap C^{1}\left(\bar{D}, M_{n}(\mathbb{C})\right)\right)$ satisfying

$$
\begin{gathered}
-\Delta u-2 i \sum_{j=1}^{d} A_{j}(x) \partial_{x_{j}} u+V(x) u=E u, \quad x \in D, \\
-\Delta u^{0}+V^{0}(x) u^{0}=E u^{0}, \quad x \in D,
\end{gathered}
$$

where $u^{0}$ also satisfies

$$
V^{0}(x) u^{0}(x)=u^{0}(x) V^{0}(x), \quad x \in D
$$

Identity (3.1) for the case when $n=1, A_{1} \equiv 0, \ldots, A_{d} \equiv 0$ first appeared in Ref. [Al]. It was generalized to the case when $n \geq 2, A_{1} \equiv 0, \ldots, A_{d} \equiv 0$ in Ref. [NS1].

Identity 3.1 can be deduced from the second Green formula. More precisely, 
formula (3.1) follows from the following chain of equalities:

$$
\begin{gathered}
\int_{D} u^{0}(x)\left(-2 i \sum_{j=1}^{d} A_{j}(x) \partial_{x_{j}}+V(x)-V^{0}(x)\right) u(x) d x \\
\underline{\underline{3.2 p, \sqrt{3.4}}} \int_{D}\left(u^{0}(x)(\Delta+E) u(x)-V^{0}(x) u^{0}(x) u(x)\right) d x \\
\underline{\underline{3.3}} \int_{D}\left(u^{0}(x) \Delta u(x)-\Delta u^{0}(x) u(x)\right) d x \\
=\int_{\partial D} u^{0}(x)\left(\Phi(E)-\Phi_{V^{0}}(E)\right)\left(\left.u\right|_{\partial D}\right)(x) d x \\
+\int_{\partial D}\left(u^{0}(x) \Phi_{V^{0}}(E)\left(\left.u\right|_{\partial D}\right)(x)-\Phi_{V^{0}}(E)\left(\left.u^{0}\right|_{\partial D}\right)(x) u(x)\right) d x \\
=\int_{\partial D} u^{0}(x)\left(\Phi(E)-\Phi_{V^{0}}(E)\right)\left(\left.u\right|_{\partial D}\right)(x) d x \\
+\int_{D}\left(u^{0}(x) V^{0}(x) \widetilde{u}(x)-V^{0}(x) u^{0}(x) \widetilde{u}(x)\right) d x \\
\underline{\underline{3.4}} \int_{\partial D} u^{0}(x)\left(\Phi(E)-\Phi_{V^{0}}(E)\right)\left(\left.u\right|_{\partial D}\right)(x) d x,
\end{gathered}
$$

where $\widetilde{u}$ satisfies $(3.3)$ and $\left.\widetilde{u}\right|_{\partial D}=\left.u\right|_{\partial D}$.

\subsection{Symmetries of functions $\psi^{0}, \psi_{\gamma}^{0}, \psi^{+, 0}$ and $R^{0}, R_{\gamma}^{0}, R^{+, 0}$}

We denote by $L_{c}^{\infty}\left(\mathbb{R}^{d}\right)$ the set of compactly supported functions from $L^{\infty}\left(\mathbb{R}^{d}\right)$.

Lemma 3.1. Let $V^{0} \in L_{c}^{\infty}\left(\mathbb{R}^{d}\right)$ satisfy either 2.15) or 2.16). Then the following identities hold:

$$
\begin{aligned}
V^{0}(x) \psi^{0}(x, k) & =\psi^{0}(x, k) V^{0}(x), \\
V^{0}(x) R^{0}(x, y, k) & =R^{0}(x, y, k) V^{0}(x), \\
R^{0}(x, y, k) & =R^{0}(y, x,-k),
\end{aligned}
$$

where $x, y \in \mathbb{R}^{d}, x \neq y, k \in \mathbb{C}^{d} \backslash\left(\mathbb{R}^{d} \cup \mathcal{E}_{V^{0}}\right)$.

Proof. Let $k \in \mathbb{C}^{d} \backslash\left(\mathbb{R}^{d} \cup \mathcal{E}_{V^{0}}\right)$ be fixed. Then equation (1.11) with $A_{1} \equiv 0, \ldots$, $A_{d} \equiv 0, V \equiv V^{0}$ is uniquely solvable for $\psi^{0}=e^{i k x} \mu^{0}$ with $\mu^{0} \in L^{\infty}\left(\mathbb{R}^{d}, M_{n}(\mathbb{C})\right)$.

Suppose, first, that $(2.15)$ holds. In this case it follows from formula (1.11) that $\psi^{0}(x, k)$ is a diagonal matrix for all $x \in \mathbb{R}^{d}$. Hence $(3.5)$ holds.

Suppose now that 2.16 holds, so that $V^{0}(x)=\mathcal{V}^{0} v^{0}(x), x \in \mathbb{R}^{d}$. Let $U$ be a non-degenerated $n \times n$ matrix such that

$$
U \mathcal{V}^{0} U^{-1} \stackrel{\text { def }}{=} \Lambda=\left[\begin{array}{ccc}
\Lambda_{1} & \cdots & 0 \\
\vdots & \ddots & \vdots \\
0 & \cdots & \Lambda_{s}
\end{array}\right], \quad \Lambda_{j}=\left[\begin{array}{ccccc}
\lambda_{j} & 1 & 0 & \cdots & 0 \\
0 & \lambda_{j} & 1 & \cdots & 0 \\
\vdots & \vdots & \vdots & \ddots & \vdots \\
0 & 0 & 0 & \cdots & 1 \\
0 & 0 & 0 & \cdots & \lambda_{j}
\end{array}\right]
$$


where $\Lambda_{j} \in M_{n_{j}}(\mathbb{C}), j=1, \ldots, s$. Define $\psi^{\prime}=U \psi^{0} U^{-1}$. Then $\psi^{\prime}$ satisfies the equation

$$
\psi^{\prime}(x, k)=e^{i k x} \operatorname{Id}_{n}+\int_{\mathbb{R}^{d}} G(x-y, k) \Lambda v^{0}(y) \psi^{\prime}(y, k) d y .
$$

Since the solution to 3.8$)$ is unique it follows that $\psi^{\prime}$ has the block-diagonal form:

$$
\psi^{\prime}=\left[\begin{array}{ccc}
\psi_{1}^{\prime} & \cdots & 0 \\
\vdots & \ddots & \vdots \\
0 & \cdots & \psi_{s}^{\prime}
\end{array}\right]
$$

where $\psi_{j}^{\prime} \in M_{n_{j}}(\mathbb{C}), j=1, \ldots, s$. Hence the following equations hold and have the unique solutions:

$$
\psi_{j}^{\prime}(x, k)=e^{i k x} \operatorname{Id}_{n_{j}}+\int_{\mathbb{R}^{d}} G(x-y, k) \Lambda_{j} v^{0}(y) \psi_{j}^{\prime}(y, k) d y,
$$

where $j=1, \ldots, s$.

We write $\psi_{j, i l}^{\prime}$ for the element in position $(i, l)$ in matrix $\psi_{j}^{\prime}$. Fix $j$ and consider the last row of matrix equation (3.9). We have the following equations:

$$
\psi_{j, i l}^{\prime}(x, k)=\lambda_{j} \int_{\mathbb{R}^{d}} G(x-y, k) v^{0}(y) \psi_{j, i l}^{\prime}(y, k) d y, \quad i=n_{j}, \quad l<n_{j} .
$$

We claim that equation $(3.10)$ has only the trivial solution. Suppose that, on the contrary, there is a nontrivial solution $\phi$ to equation $(3.10)$. Then we can construct a solution $\widetilde{\psi}_{j}^{\prime}$ to 3.9 different from $\psi_{j}^{\prime}$, putting $\vec{\psi}_{j, 11}^{\prime}=\psi_{j, 11}^{\prime}+\phi$ and $\widetilde{\psi}_{j, i l}^{\prime}=\psi_{i, i l}^{\prime}$ for all other $i, l$. This is a contradiction since we showed that equation $(3.9)$ has the unique solution. This shows that equation 3.10$)$ has only the trivial solution and $\psi_{j, i l}^{\prime} \equiv 0$ for $i=n_{j}$ and $l<n_{j}$.

Writing the equation (3.9) componentwise for rows with numbers $i=n_{j}-1$, $\ldots, 2$ we show by induction that $\psi_{j, i l}^{\prime} \equiv 0$ for $i>l$.

Fix $i, l$ with $i \neq l$. Subtracting equation $(3.9)$ for the element in position $(l, l)$ from equation $(3.9)$ for the element in position $(i, i)$ we get the equation

$$
\psi_{j, i i}^{\prime}(x, k)-\psi_{j, l l}^{\prime}(x, k)=\lambda_{j} \int_{\mathbb{R}^{d}} G(x-y, k) v^{0}(y)\left(\psi_{j, i i}^{\prime}(y, k)-\psi_{j, l l}^{\prime}(y, k)\right) d y .
$$

Since equation 3.10 has only the trivial solution, it follows that $\psi_{j, i i}^{\prime} \equiv \psi_{j, l l}^{\prime}$.

Now fix $i, l$ with $i \neq l, i>1, l>1$. Write equation (3.9) for the elements in positions $(i-1, i)$ and $(l-1, l)$ and subtract one from another. This leads to equation

$$
\begin{gathered}
\psi_{j, i-1, i}^{\prime}(x, k)-\psi_{j, l-1, l}^{\prime}(x, k)=\lambda_{j} \int_{\mathbb{R}^{d}} G(x-y, k) \times \\
\times v^{0}(y)\left(\psi_{j, i-1, i}^{\prime}(y, k)-\psi_{j, l-1, l}^{\prime}(y, k)\right) d y+\psi_{j, i i}^{\prime}(x, k)-\psi_{j, l l}^{\prime}(x, k) .
\end{gathered}
$$


But we showed that $\psi_{j, i i}^{\prime} \equiv \psi_{j, l l}^{\prime}$ and that equation 3.10 has only the trivial solution. This implies that $\psi_{j, i-1, i}^{\prime} \equiv \psi_{j, l-1, l}^{\prime}$. form:

Proceeding inductively, we obtain that $\psi_{j}^{\prime}$ has the following upper triangular

$$
\psi_{j}^{\prime}=\left[\begin{array}{cccccc}
\psi_{j, 11}^{\prime} & \psi_{j, 12}^{\prime} & \psi_{j, 13}^{\prime} & \cdots & \psi_{j, 1, n-1}^{\prime} & \psi_{j, 1 n}^{\prime} \\
0 & \psi_{j, 11}^{\prime} & \psi_{j, 12}^{\prime} & \cdots & \psi_{j, 1, n-2}^{\prime} & \psi_{j, 1, n-1}^{\prime} \\
\vdots & \vdots & \vdots & \vdots & \vdots & \vdots \\
0 & 0 & 0 & \cdots & \psi_{j, 11}^{\prime} & \psi_{j, 12}^{\prime} \\
0 & 0 & 0 & \cdots & 0 & \psi_{j, 11}^{\prime}
\end{array}\right] .
$$

It follows that $\psi_{j}^{\prime}(x, k)$ commutes with $\Lambda_{j}$ for all $x \in \mathbb{R}^{d}, j=1, \ldots, s$. Hence $\psi^{\prime}(x, k)$ commutes with $\Lambda$ and $\psi(x, k)$ commutes with $\mathcal{V}^{0}$ for all $x \in \mathbb{R}^{d}$. Property (3.5) is proved.

Formula $(3.6)$ can be proved in a similar way.

The proof of (3.7) for the case when $n=1$ was given in [No3]. This proof also works in the case when $n \geq 2$ if either (2.15) or (2.16) holds.

Remark 3.1. In a similar way with Lemma 3.1 it can be proved that if $V^{0} \in$ $L_{c}^{\infty}\left(\mathbb{R}^{d}\right)$ satisfies either 2.15$)$ or 2.16 , then the following formulas hold:

$$
\begin{aligned}
V^{0}(x) \psi_{\gamma}^{0}(x, k) & =\psi_{\gamma}^{0}(x, k) V^{0}(x), \\
V^{0}(x) R_{\gamma}^{0}(x, y, k) & =R_{\gamma}^{0}(x, y, k) V^{0}(x), \\
R_{\gamma}^{0}(x, y, k) & =R_{-\gamma}^{0}(y, x,-k),
\end{aligned}
$$

where $\gamma \in S^{d-1}, x, y \in \mathbb{R}^{d}, x \neq y, k \in \mathbb{R}^{d} \backslash\left(0 \cup \mathcal{E}_{V^{0}, \gamma}\right)$;

$$
\begin{aligned}
V^{0}(x) \psi^{+, 0}(x, k) & =\psi^{+, 0}(x, k) V^{0}(x), \\
V^{0}(x) R^{+, 0}(x, y, k) & =R^{+, 0}(x, y, k) V^{0}(x), \\
R^{+, 0}(x, y, k) & =R^{+, 0}(y, x,-k),
\end{aligned}
$$

where $x, y \in \mathbb{R}^{d}, x \neq y, k \in \mathbb{R}^{d} \backslash\left(0 \cup \mathcal{E}_{V^{0}}^{+}\right)$.

Lemma 3.2. Let $V^{0} \in L_{c}^{\infty}\left(\mathbb{R}^{d}\right)$. Then:

1. if $k, l \in \mathbb{C}^{d} \backslash \mathbb{R}^{d}, k^{2}=l^{2}, \operatorname{Im} k=\operatorname{Im} l, k \notin \mathcal{E}_{V^{0}}$, then $l \notin \mathcal{E}_{V^{0}}$ and the following formula holds:

$$
R^{0}(x, y, k)=R^{0}(x, y, l)
$$

where $x, y \in \mathbb{R}^{d}, x \neq y$;

2. if $k, l \in \mathbb{R}^{d} \backslash 0, k^{2}=l^{2}, k \notin \mathcal{E}_{V^{0}}^{+}$, then $l \notin \mathcal{E}_{V^{0}}^{+}$and the following formula holds:

$$
R^{+, 0}(x, y, k)=R^{+, 0}(x, y, l),
$$

where $x, y \in \mathbb{R}^{d}, x \neq y$. 
Proof. Part 1 follows from 2.17) and from the following formula of Ref. [HN1]:

$$
G(x, k)=G(x, l), \quad x \in \mathbb{R}^{d} \backslash 0, k, l \in \mathbb{C}^{d} \backslash \mathbb{R}^{d}, k^{2}=l^{2}, \operatorname{Im} k=\operatorname{Im} l .
$$

Part 2 follows from formulas (2.17), 2.19) and from identity

$$
G^{+}(x, k)=G^{+}(x, l), \quad x \in \mathbb{R}^{d} \backslash 0, k, l \in \mathbb{R}^{d} \backslash 0, k^{2}=l^{2},
$$

which is a consequence of the following well-known explicit formulas:

$$
\begin{aligned}
G^{+}(x, k) & =-\frac{i}{4} H_{0}^{(1)}(|k||x|), \quad d=2, \\
G^{+}(x, k) & =-\frac{e^{i|k||x|}}{4 \pi|x|}, \quad d=3,
\end{aligned}
$$

where $H_{0}^{(1)}$ is the Hankel function of the first kind.

\subsection{Reformulation of equations (1.8), (1.11) and formulas} (1.10), 1.13) in terms of background coefficients

Subtracting equation (1.11) written for $\psi$ from equation 1.11 written for $\psi^{0}$ we obtain formula

$$
\begin{aligned}
& \psi(x, k)-\psi^{0}(x, k)-\int_{\mathbb{R}^{d}} G(x-y, k) V^{0}(y)\left(\psi(y, k)-\psi^{0}(y, k)\right) d y \\
& =\int_{\mathbb{R}^{d}} G(x-y, k)\left(-2 i \sum_{j=1}^{d} A_{j}(y) \partial_{y_{j}}+V(y)-V^{0}(y)\right) \psi(y, k) d y,
\end{aligned}
$$

where $x \in \mathbb{R}^{d}, k \in \mathbb{C}^{d} \backslash\left(\mathbb{R}^{d} \cup \mathcal{E} \cup \mathcal{E}_{V^{0}}\right)$. Comparing this equation with 2.17), we obtain

$$
\begin{gathered}
\psi(x, k)=\psi^{0}(x, k)+\int_{\mathbb{R}^{d}} R^{0}(x, y, k) \times \\
\times\left(-2 i \sum_{j=1}^{d} A_{j}(y) \partial_{y_{j}}+V(y)-V^{0}(y)\right) \psi(y, k) d y,
\end{gathered}
$$

where $x \in \mathbb{R}^{d}, k \in \mathbb{C}^{d} \backslash\left(\mathbb{R}^{d} \cup \mathcal{E} \cup \mathcal{E}_{V^{0}}\right)$.

Similarly, we can obtain the following equations:

$$
\begin{gathered}
\psi_{\gamma}(x, k)=\psi_{\gamma}^{0}(x, k)+\int_{\mathbb{R}^{d}} R_{\gamma}^{0}(x, y, k) \times \\
\times\left(-2 i \sum_{j=1}^{d} A_{j}(y) \partial_{y_{j}}+V(y)-V^{0}(y)\right) \psi_{\gamma}^{+}(y, k) d y,
\end{gathered}
$$


where $\gamma \in S^{d-1}, x \in \mathbb{R}^{d}, k \in \mathbb{R}^{d} \backslash\left(0 \cup \mathcal{E}_{\gamma} \cup \mathcal{E}_{V^{0}, \gamma}\right)$;

$$
\begin{gathered}
\psi^{+}(x, k)=\psi^{+, 0}(x, k)+\int_{\mathbb{R}^{d}} R^{+, 0}(x, y, k) \times \\
\times\left(-2 i \sum_{j=1}^{d} A_{j}(y) \partial_{y_{j}}+V(y)-V^{0}(y)\right) \psi^{+}(y, k) d y,
\end{gathered}
$$

where $x \in \mathbb{R}^{d}, k \in \mathbb{R}^{d} \backslash\left(0 \cup \mathcal{E}^{+} \cup \mathcal{E}_{V^{0}}^{+}\right)$.

From formula 3.19 with $A_{1} \equiv 0, \ldots, A_{d} \equiv 0, V \equiv 0$ and from formulas (3.6), (3.7), (3.17) we obtain

$$
e^{-i l x} \operatorname{Id}_{n}=\psi^{0}(x,-l)-\int_{\mathbb{R}^{d}} e^{-i l y} V^{0}(y) R^{0}(y, x, k) d y,
$$

where $x \in \mathbb{R}^{d}, k, l \in \mathbb{C}^{d} \backslash \mathbb{R}^{d}, k^{2}=l^{2}, \operatorname{Im} k=\operatorname{Im} l, k \notin \mathcal{E}_{V^{0}}$.

Further, we have the following chain of equalities:

$$
\begin{gathered}
(2 \pi)^{d} h(k, l) \stackrel{\sqrt{1.13}}{=} \int_{\mathbb{R}^{d}} e^{-i l x} V^{0}(x) \psi(x, k) d x \\
+\int_{\mathbb{R}^{d}} e^{-i l x}\left(-2 i \sum_{j=1}^{d} A_{j}(x) \partial_{x_{j}}+V(x)-V^{0}(x)\right) \psi(x, k) d x \\
\stackrel{\underline{3.22}}{=} \int_{\mathbb{R}^{d}} e^{-i l x} V^{0}(x) \psi(x, k) d x+\int_{\mathbb{R}^{d}} \psi^{0}(x,-l) \times \\
\times\left(-2 i \sum_{j=1}^{d} A_{j}(x) \partial_{x_{j}}+V(x)-V^{0}(x)\right) \psi(x, k) d x \\
\quad-\int_{\mathbb{R}^{d}} e^{-i l y} V^{0}(y) \int_{\mathbb{R}^{d}} R^{0}(y, x, k) \times \\
\times\left(-2 i \sum_{j=1}^{d} A_{j}(x) \partial_{x_{j}}+V(x)-V^{0}(x)\right) \psi(x, k) d x d y,
\end{gathered}
$$

where $k, l \in \mathbb{C}^{d} \backslash \mathbb{R}^{d}, k^{2}=l^{2}, \operatorname{Im} k=\operatorname{Im} l, k \notin \mathcal{E} \cup \mathcal{E}_{V^{0}}$.

From this formula and formula (3.19) it follows that

$$
\begin{gathered}
h(k, l)=h^{0}(k, l)+(2 \pi)^{-d} \int_{\mathbb{R}^{d}} \psi^{0}(x,-l) \times \\
\times\left(-2 i \sum_{j=1}^{d} A_{j}(x) \partial_{x_{j}}+V(x)-V^{0}(x)\right) \psi(x, k) d x,
\end{gathered}
$$

where $k, l \in \mathbb{C}^{d} \backslash \mathbb{R}^{d}, k^{2}=l^{2}, \operatorname{Im} k=\operatorname{Im} l, k \notin \mathcal{E} \cup \mathcal{E}_{V^{0}}$. 
In a similar way with formula $(3.23)$, using formulas 3.21$),(3.15),(3.16)$, (3.18) instead of (3.19), (3.6), 3.7), (3.17), we can obtain formula

$$
\begin{gathered}
f(k, l)=f^{0}(k, l)+(2 \pi)^{-d} \int_{\mathbb{R}^{d}} \psi^{+, 0}(x,-l) \times \\
\times\left(-2 i \sum_{j=1}^{d} A_{j}(x) \partial_{x_{j}}+V(x)-V^{0}(x)\right) \psi^{+}(x, k) d x,
\end{gathered}
$$

where $k, l \in \mathbb{R}^{d} \backslash 0, k^{2}=l^{2}, k \notin \mathcal{E}^{+} \cup \mathcal{E}_{V^{0}}^{+}$.

\subsection{The final part of the proof of Theorem 2.1}

It follows from formulas $(3.5),(3.14)$ that we can apply identity $(3.1)$ to $(3.23)$ and (3.24). Applying (3.1) to (3.23) and (3.24), we obtain (2.25) and (2.31), respectively.

We recall that function $G(x, k)$ satisfies the following equation at fixed $k \in$ $\mathbb{C}^{d} \backslash \mathbb{R}^{d}$, see, e.g., [Fa1]:

$$
\Delta_{x} G(x, k)+k^{2} G(x, k)=\delta(x) .
$$

It follows from formulas (2.17), 2.20), 2.23), 3.25) that $R^{0}(x, y, k)$ at fixed $k \in \mathbb{C}^{d} \backslash\left(\mathbb{R}^{d} \cup \mathcal{E}_{V^{0}}\right)$, satisfies the equations:

$$
\begin{aligned}
& \left(\Delta_{x}-V^{0}(x)+k^{2}\right) R(x, y, k)=\delta_{y}(x) \operatorname{Id}_{n}, \quad \text { at fixed } y \in \mathbb{R}^{d}, \\
& \left(\Delta_{y}-V^{0}(y)+k^{2}\right) R(x, y, k)=\delta_{x}(y) \operatorname{Id}_{n}, \quad \text { at fixed } x \in \mathbb{R}^{d} .
\end{aligned}
$$

Taking this into account, using formula (3.6), applying identity (3.1) to 3.19) with $x \notin \bar{D}$ and passing to the limit in the resulting formula as $x$ tends to a point at $\partial D$, we obtain formula $(2.26)$. Formulas $(2.29)$ and 2.32 can be obtained in a similar way, if we use equations $(3.20)$ and $(3.21)$ instead of $(3.19)$.

We will prove formula $(2.28)$ using the ideas of Ref. [No4]. Note that formula (2.24) can be written in the form

$$
\begin{gathered}
e^{i l x} \operatorname{Id}_{n}-\widetilde{\psi}_{\gamma}^{0}(x, k, l)-\int_{\mathbb{R}^{d}} G_{\gamma}(x-y, k) V^{0}(y)\left(e^{i l y}-\widetilde{\psi}_{\gamma}^{0}(y, k, l)\right) d y \\
=-\int_{\mathbb{R}^{d}} G(x-y, k) V^{0}(y) e^{i l y} d y
\end{gathered}
$$

From formulas 2.10), 2.17), 2.18) it follows that function $R_{\gamma}^{0}(\cdot, y, k)$ is welldefined at fixed $y \in \mathbb{R}^{d}, k \in \mathbb{R}^{d} \backslash\left(0 \cup \mathcal{E}_{V^{0}, \gamma}\right)$ and satisfies

$$
R_{\gamma}^{0}(x, y, k)=G_{\gamma}(x-y, k)+\int_{\mathbb{R}^{d}} G_{\gamma}(x-z, k) V^{0}(z) R_{\gamma}^{0}(z, y, k) d z,
$$


where $x, y \in \mathbb{R}^{d}, \gamma \in S^{d-1}, k \in \mathbb{R}^{d} \backslash\left(0 \cup \mathcal{E}_{V^{0}, \gamma}\right)$.

Comparing (3.27) with (3.28), we obtain the following formula:

$$
e^{i l x} \operatorname{Id}_{n}=\widetilde{\psi}_{\gamma}^{0}(x, k, l)-\int_{\mathbb{R}^{d}} R_{\gamma}^{0}(x, y, k) V^{0}(y) e^{i l y} d y,
$$

where $x \in \mathbb{R}^{d}, k \in \mathbb{R}^{d} \backslash\left(0 \cup \mathcal{E}_{V^{0}, \gamma}\right), l \in \mathbb{R}^{d}, k^{2}=l^{2}, \gamma \in S^{d-1}$.

Replacing $k, l, \gamma$ by $-k,-l,-\gamma$ in formula (3.29) and using formulas (3.12), (3.13, we obtain the equality

$$
e^{-i l x} \operatorname{Id}_{n}=\widetilde{\psi}_{-\gamma}^{0}(x,-k,-l)-\int_{\mathbb{R}^{d}} e^{-i l y} V^{0}(y) R_{\gamma}^{0}(y, x, k) d y,
$$

where $x \in \mathbb{R}^{d}, k, l \in \mathbb{R}^{d} \backslash 0, k^{2}=l^{2}, k \notin \mathcal{E}_{V^{0}, \gamma}, \gamma \in S^{d-1}$.

Formula $(3.30)$ is an analog of formula (3.22). The remaining part of the proof of $(2.28)$ is similar to the proof of formula 2.25 .

\section{Proof of Theorem 2.2}

\subsection{Auxilary results}

Consider the following Dirichlet problem for function $\psi$ :

$$
\left\{\begin{array}{l}
L \psi \equiv-\Delta \psi-2 i \sum_{j=1}^{d} A_{j}(x) \partial_{x_{j}} \psi+V(x) \psi=E \psi, \quad x \in D, \\
\left.\psi\right|_{\partial D}=\varphi,
\end{array}\right.
$$

where $\varphi$ is some given function on $\partial D$.

Our goal in this subsection is to prove the following lemma.

Lemma 4.1. Let $A_{1}, \ldots, A_{d}, V \in C_{c}^{0, \alpha}\left(D, M_{n}(\mathbb{C})\right)$ for some $0<\alpha \leq 1$. Suppose that $E$ is not a Dirichlet eigenvalue for operators $L$ and $-\Delta$ in $D$. Then:

1. for any $\varphi \in C^{1, \beta}\left(\partial D, M_{n}(\mathbb{C})\right), 0<\beta<1$, there exists the unique solution $\psi \in C^{2}\left(D, M_{n}(\mathbb{C})\right) \cap C^{1}\left(\bar{D}, M_{n}(\mathbb{C})\right)$ to problem (4.1);

2. $\psi$ is the unique solution of class $C^{1}\left(\bar{D}, M_{n}(\mathbb{C})\right)$ to equation

$$
\psi(x)=\psi^{0}(x)+\int_{D} \Gamma(x, y, k)\left(-2 i \sum_{j=1}^{d} A_{j}(y) \partial_{y_{j}}+V(y)\right) \psi(y) d y,
$$

where $\Gamma(x, y, k)$ is the Green function for Dirichlet problem for operator $\Delta+E$ in $D, E=k^{2}$, and $\psi^{0} \in C^{2}\left(D, M_{n}(\mathbb{C})\right) \cap C^{1}\left(\bar{D}, M_{n}(\mathbb{C})\right)$, is the unique solution to Dirichlet problem

$$
\left\{\begin{array}{l}
\Delta \psi^{0}+E \psi^{0}=0 \quad \text { in } D \\
\left.\psi^{0}\right|_{\partial D}=\varphi
\end{array}\right.
$$


3. the operator $S: C^{1, \beta}\left(\partial D, M_{n}(\mathbb{C})\right) \rightarrow C^{1}\left(\bar{D}, M_{n}(\mathbb{C})\right), S(\varphi)=\psi$, is a continuous linear operator.

We need two other lemmas to prove Lemma 4.1.

Lemma 4.2. Let $A_{1}, \ldots, A_{d}, V \in C_{c}^{0, \alpha}\left(D, M_{n}(\mathbb{C})\right)$ for some $0<\alpha \leq 1$. Suppose that $E$ is not a Dirichlet eigenvalue for operator $L$ in $D$. Then:

1. for any $\psi^{0} \in C^{1}\left(\bar{D}, M_{n}(\mathbb{C})\right) \cap C^{2}\left(D, M_{n}(\mathbb{C})\right)$ satisfying $(\Delta+E) \psi^{0}=0$ in $D$ there exists the unique solution $\psi \in C^{1}\left(\bar{D}, M_{n}(\mathbb{C})\right)$ to equation 4.2 ;

2. $\psi$ belongs to $C^{2}\left(D, M_{n}(\mathbb{C})\right)$ and satisfies 4.1 with $\varphi=\left.\psi^{0}\right|_{\partial D}$;

3. there exists a constant $C>0$, not depending on $\psi^{0}$, such that

$$
\|\psi\|_{C^{1}(\bar{D})} \leq C\left\|\psi^{0}\right\|_{C^{1}(\bar{D})} .
$$

Proof of Lemma 4.2. 1. Reduction to a system of integral equations. We introduce the following notations:

$$
\begin{array}{lll}
\psi_{0}(x)=\psi(x), & \psi_{j}(x)=\partial_{x_{j}} \psi(x), & j=1, \ldots, d, \\
\psi_{0}^{0}(x)=\psi^{0}(x), & \psi_{j}^{0}(x)=\partial_{x_{j}} \psi^{0}(x), & j=1, \ldots, d, \\
a_{0}(x)=V(x), & a_{j}(x)=-2 i A_{j}(x), & j=1, \ldots, d, \\
\Gamma_{0}(x, y, k)=\Gamma(x, y, k), & \Gamma_{j}(x, y, k)=\partial_{x_{j}} \Gamma(x, y, k), & j=1, \ldots, d .
\end{array}
$$

Differentiating equation 4.2 we obtain the following system of coupled linear integral equations for $\psi_{j} \in C(\bar{D}), j=0, \ldots, d$ :

$$
\psi_{j}(x)=\psi_{j}^{0}(x)+\sum_{m=0}^{d} \int_{D} \Gamma_{j}(x, y, k) a_{m}(y) \psi_{m}(y) d y, \quad j=0, \ldots, d .
$$

System 4.5 can be considered as a Fredholm equation of second kind in space $\left(C\left(\bar{D}, M_{n}(\mathbb{C})\right)\right)^{d+1}$.

Suppose that functions $\psi_{j} \in C\left(\bar{D}, M_{n}(\mathbb{C})\right), j=0, \ldots, d$, solve (4.5). Denote $\psi=\psi_{0}$. The first equation of 4.5 implies that $\psi \in C^{1}\left(\bar{D}, M_{n}(\mathbb{C})\right)$. Differentiating the first equation with respect to $x_{1}, \ldots, x_{d}$, we see that $\partial_{x_{j}} \psi=\psi_{j}$. Hence $\psi$ satisfies 4.2 .

One can see that solutions $\psi$ of class $C^{1}\left(\bar{D}, M_{n}(\mathbb{C})\right)$ to 4.2 are in bijective correspondence with solutions $\psi_{j} \in C\left(\bar{D}, M_{n}(\mathbb{C})\right), j=0, \ldots, d$, to system 4.5 .

2. Smoothness of solution to (4.2). It follows from properties of fundamental solution $\Gamma$ that any solution $\psi \in C^{1}\left(\bar{D}, M_{n}(\mathbb{C})\right)$ to $(4.2)$ belongs to $C_{\text {loc }}^{1, \gamma}\left(D, M_{n}(\mathbb{C})\right)$ (the space of continuously differentiable $M_{n}(\mathbb{C})$-valued functions on $D$ with locally Hölder continuous derivatives) for any $0<\gamma<1$.

In a similar way with Lemma 4.2 , p. 55 of [GT] it can be shown then that $\psi \in C^{2}\left(D, M_{n}(\mathbb{C})\right)$ and that $\psi$ satisfies 4.1 with $\varphi=\left.\psi^{0}\right|_{\partial D}$.

3. Existence and uniqueness. As we noted in part 1 of the proof of this lemma, system of equations 4.5 can be considered as a Fredholm equation of second kind in Banach space $\left(C\left(D, M_{n}(\mathbb{C})\right)\right)^{d+1}$. To show that this equation has 
the unique solution it is sufficient to show that the corresponding homogeneous equation has only the trivial solution. But if follows from parts 1,2 of the proof that any solution to the homogeneous equation gives rise to a solution to (4.1) with zero boundary condition. Since $E$ is not a Dirichlet eigenvalue for $L$ in $D$, it follows that the homogeneous equation corresponding to 4.5 can have only the trivial solution.

Hence, using part 1 of the proof, we obtain that system 4.5 has the unique solution and this solution gives rise to the unique solution $\psi \in C^{1}\left(\bar{D}, M_{n}(\mathbb{C})\right)$ to 4.2 . By part 2 of the proof this function $\psi$ belongs to $C^{2}\left(D, M_{n}(\mathbb{C})\right)$ and satisfies 4.1 with $\varphi=\left.\psi^{0}\right|_{\partial D}$.

Property 3 follows from Fredholm alternative for system 4.5 which asserts the existence of continuous inverse for Fredholm operator of (4.5).

Lemma 4.3. Suppose that $E$ is not a Dirichlet eigenvalue for operator $-\Delta$ in $D$. Then for any $\varphi \in C^{1, \beta}\left(\partial D, M_{n}(\mathbb{C})\right), 0<\beta<1$, there exists the unique $\psi^{0} \in$ $C^{2}\left(D, M_{n}(\mathbb{C})\right) \cap C^{1}\left(\bar{D}, M_{n}(\mathbb{C})\right)$ solving Dirichlet problem 4.3). Furthermore, there exists such $C_{\beta}>0$, not depending on $\varphi$, that

$$
\left\|\psi^{0}\right\|_{C^{1}(\bar{D})} \leq C_{\beta}\|\varphi\|_{C^{1, \beta}(\partial D)} .
$$

Proof of Lemma 4.3. The solution is unique since $E$ is not a Dirichlet eigenvalue for $-\Delta$. To show existence, we can reduce problem (4.1) to a corresponding Fredholm integral equation of second kind as in Lemma 4.2 .

Existence of such $C_{\beta}>0$ that 4.6 holds follows from Lemmas 2.16, 2.23 of $[\mathrm{CK}]$.

Proof of Lemma 4.1. One can see that solution to (4.1) is unique since $E$ is not a Dirichlet eigenvalue for $L$ in $D$.

Now let $\psi^{0}$ be the unique solution to 4.3 constructed in Lemma 4.3 . Then Lemma 4.2 gives us the solution to 4.1 with desired properties.

Property 3 is a consequence of formulas 4.4, 4.6.

\subsection{Compactness of integral operators in equations (2.26),} (2.29), 2.32)

In this subsection we show that the integral operator in equation $(2.26)$ is a compact linear operator on $C^{1, \beta}\left(\partial D, M_{n}(\mathbb{C})\right)$ for any fixed $0<\beta<1$. The case of operators in equations 2.29, 2.32 can be considered in a similar way.

Throughout this subsection we assume that $k \in \mathbb{C}^{d} \backslash\left(\mathbb{R}^{d} \cup \mathcal{E}_{V^{0}}\right)$ is fixed.

Rewrite equation $(2.26)$ in the following form:

$$
\psi=\psi^{0}+R^{0}(k)\left(\Phi(E)-\Phi_{V^{0}}(E)\right) \psi,
$$

where $R^{0}(k)$ is the integral operator with Schwartz kernel $R^{0}(x, y, k), x, y \in \partial D$.

We are going to show that $R^{0}(k)$ is a linear continuous operator on the space $C^{1, \beta}\left(\partial D, M_{n}(\mathbb{C})\right)$. To show this we use the representation 2.20 . 
It follows from Theorems 2.12, 2.17 of [CK] that the integral operator on $C^{1, \beta}\left(\partial D, M_{n}(\mathbb{C})\right)$ with Schwartz kernel $G(x-y, k), x, y \in \partial D$, is continuous.

To see that the integral operator with Schwartz kernel $e^{i k(x-y)} r^{0}(x, y, k), x$, $y \in \partial D$, is continuous on $C^{1, \beta}\left(\partial D, M_{n}(\mathbb{C})\right)$, we will use formula (2.21).

It follows from 2.21, 2.22) (if we take into account that $V^{0}$ has a compact support in $D)$ that derivatives $\partial_{x_{i}} r^{0}(x, y, k), \partial_{x_{i}} \partial_{x_{j}} r^{0}(x, y, k), i, j=1, \ldots$, $d$, exist and are continuous for $x, y$ belonging to some neighborhood of set $\partial D$. Hence the operator with Schwartz kernel $e^{i k(x-y)} r^{0}(x, y, k), x, y \in \partial D$, is continuous on $C^{1, \beta}\left(\partial D, M_{n}(\mathbb{C})\right)$. Hence $R^{0}(k)$ is also a linear continuous operator on $C^{1, \beta}\left(\partial D, M_{n}(\mathbb{C})\right)$.

If we show that $\Phi(E)-\Phi_{V^{0}}(E)$ is a compact operator on $C^{1, \beta}\left(\partial D, M_{n}(\mathbb{C})\right)$, formula (4.7) will imply that integral operator in formula (2.26) is compact.

Let $S$ be the operator defined in part 3 of Lemma 4.1. Define $S_{V^{0}}$ by the same formula using coefficients $A_{1}^{0} \equiv 0, \ldots, A_{d}^{0} \equiv 0, V^{0}$ in 4.2 .

It follows from Lemma 4.1 , part 3 , that $S, S_{V^{0}}$ are linear continuous operators from $C^{1, \beta}\left(\partial D, M_{n}(\mathbb{C})\right)$ to $C^{1}\left(\bar{D}, M_{n}(\mathbb{C})\right)$.

Taking into account equation 4.2 , we obtain the following formula:

$$
\Phi(E)-\Phi_{V^{0}}(E)=N S-N_{V^{0}} S_{V^{0}},
$$

where $N, N_{V^{0}}$ are the linear continuous operators acting from $C^{1}\left(\bar{D}, M_{n}(\mathbb{C})\right)$ to $C^{2}\left(\partial D, M_{n}(\mathbb{C})\right)$ defined by the following formulas:

$$
\begin{gathered}
(N \psi)(x)=\int_{D} \frac{\partial \Gamma}{\partial \nu_{x}}(x, y, k)\left(-2 i \sum_{j=1}^{d} A_{j}(y) \partial_{y_{j}}+V(y)\right) \psi(y) d y \\
\left(N_{V^{0}} \psi\right)(x)=\int_{D} \frac{\partial \Gamma}{\partial \nu_{x}}(x, y, k) V^{0}(y) \psi(y) d y
\end{gathered}
$$

where $\Gamma$ is the Green function for Dirichlet problem for operator $\Delta+E$ in $D$ and $\nu_{x}$ denotes the unit exterior normal to $\partial D$ at $x \in \partial D$.

Taking into accout that inclusion $C^{2}\left(\partial D, M_{n}(\mathbb{C})\right) \hookrightarrow C^{1, \beta}\left(\partial D, M_{n}(\mathbb{C})\right)$ is compact, we obtain that $N, N_{V^{0}}$ are compact operators from $C^{1}\left(\bar{D}, M_{n}(\mathbb{C})\right)$ to

$C^{1, \beta}\left(\partial D, M_{n}(\mathbb{C})\right)$. It follows from continuity of $S, S_{V^{0}}$, from compactness of $N, N_{V^{0}}$ and from formula 4.8 that $\Phi(E)-\Phi_{V^{0}}(E)$ is a compact operator on $C^{1, \beta}\left(\partial D, M_{n}(\mathbb{C})\right)$.

Now formula 4.7 implies that the integral operator in equation 2.26 is compact on $C^{1, \beta}\left(\overline{\partial D}, M_{n}(\mathbb{C})\right)$.

In a similar way it can be shown that the integral operators in equations (2.29), 2.32 are compact.

\subsection{Unique solvability of equations 2.26, 2.29, 2.32}

In this subsection we will finish the proof of the part 1 of Theorem 2.2], The proof of parts 2, 3 of Theorem 2.2 can be finished in a similar way.

In fact, in this subsection we will prove the following lemma. 
Lemma 4.4. Let $k \in \mathbb{C}^{d} \backslash\left(\mathbb{R}^{d} \cup \mathcal{E}_{V^{0}}\right), k^{2}=E$, and $0<\beta<1$ be fixed. Suppose that assumptions of Theorem 2.1] are fulfilled. Then equation (2.26) is uniquely solvable for $\psi \in C^{1, \beta}\left(\partial D, M_{n}(\mathbb{C})\right)$ if and only if equation $(3.19)$ is uniquely solvable for $\psi(x, k)=e^{i k x} \mu(x, k)$ with $\mu \in W^{1, \infty}\left(\mathbb{R}^{d}, M_{n}(\mathbb{C})\right)$.

Lemma 4.4 implies the statement of part 1 of Theorem 2.2 since equation (3.19) at fixed $k \in \mathbb{C}^{d} \backslash\left(\mathbb{R}^{d} \cup \mathcal{E}_{V^{0}}\right)$ is uniquely solvable for $\psi(x, k)=e^{i k x} \mu(x, k)$ with $\mu \in W^{1, \infty}\left(\mathbb{R}^{d}, M_{n}(\mathbb{C})\right)$ if and only if $k \notin \mathcal{E}$.

Before passing to the proof of Lemma 4.4 note that function $\psi^{0}(x, k)$ of 2.26 is defined as the solution to (1.11) with coefficients $A_{1}^{0} \equiv 0, \ldots, A_{d}^{0} \equiv 0, V^{0}$, such that $\psi^{0}(x, k)=e^{i k x} \mu^{0}(x, k), \mu^{0} \in W^{1, \infty}\left(\mathbb{R}^{d}, M_{n}(\mathbb{C})\right)$. Note that function $\psi^{0}(x, k)$ belongs to $C^{2}\left(\mathbb{R}^{d}, M_{n}(\mathbb{C})\right)$ and satisfies the equation

$$
-\Delta \psi^{0}+V^{0}(x) \psi^{0}=E \psi^{0}, \quad x \in \mathbb{R}^{d} .
$$

The proof of this fact is similar to the proof of parts 1,2 of Lemma 4.2 .

We will prove Lemma 4.4 in two steps.

1. Extending a solution to equation $(2.26)$ to a solution to equation $(3.19)$. Let $\varphi \in C^{1, \beta}\left(D, M_{n}(\mathbb{C})\right), 0<\beta<1$, be a solution to equation (2.26). We will show that $\varphi$ gives rise to a solution $\psi(x, k)=e^{i k x} \mu(x, k), \mu \in W^{1, \infty}\left(\mathbb{R}^{d}, M_{n}(\mathbb{C})\right)$ to equation $(3.19)$.

Let $\varphi^{+} \in C^{2}\left(D, M_{n}(\mathbb{C})\right) \cap C^{1}\left(\bar{D}, M_{n}(\mathbb{C})\right)$ be the unique solution to 4.1 given by Lemma 4.1. Define $\varphi^{-}$by formula

$$
\varphi^{-}(x)=\psi^{0}(x, k)+\int_{\partial D} A^{0}(x, y, k) \varphi(y) d y, \quad x \in \mathbb{R}^{d} \backslash D .
$$

Using formulas (2.26), 2.27), 3.1), we obtain the following formula:

$$
\begin{gathered}
\int_{D} R^{0}(x, y, k)\left(-2 i \sum_{j=1}^{d} A_{j}(y) \partial_{y_{j}}+V(y)-V^{0}(y)\right) \varphi^{+}(y) d y \\
=\int_{\partial D} A(x, y, k) \varphi(y) d y
\end{gathered}
$$

where $x \in \mathbb{R}^{d} \backslash \bar{D}$. It follows from 2.20, 2.22 that formula 4.11) holds for $x \in \mathbb{R}^{d} \backslash D$. Using formulas 4.10, (4.11), we obtain formula

$$
\begin{gathered}
\varphi^{-}(x)=\psi^{0}(x, k)+\int_{D} R^{0}(x, y, k) \times \\
\times\left(-2 i \sum_{j=1}^{d} A_{j}(y) \partial_{y_{j}}+V(y)-V^{0}(y)\right) \varphi^{+}(y) d y,
\end{gathered}
$$

where $x \in \mathbb{R}^{d} \backslash D$. It follows from 2.20, (2.21), 2.22), 4.12) that $\varphi^{-} \in$ $C^{1}\left(\mathbb{R}^{d} \backslash D, M_{n}(\mathbb{C})\right)$. 
Note that the following formula holds:

$$
\begin{gathered}
\int_{D} R^{0}(x, y, k)\left(-2 i \sum_{j=1}^{d} A_{j}(y) \partial_{y_{j}}+V(y)-V^{0}(y)\right) \varphi^{+}(y) d y \\
=\int_{D} \delta_{x}(y) \varphi^{+}(y) d y+\int_{\partial D}\left(R^{0}(x, y, k) \frac{\partial \varphi^{+}(y)}{\partial \nu_{y}}-\frac{\partial R^{0}}{\partial \nu_{y}}(x, y, k) \varphi(y)\right) d y,
\end{gathered}
$$

where $x \notin \partial D$.

Formula 4.13 follows from the following chain of equalities:

$$
\begin{gathered}
\int_{D} R^{0}(x, y, k)\left(-2 i \sum_{j=1}^{d} A_{j}(y) \partial_{y_{j}}+V(y)-V^{0}(y)\right) \varphi^{+}(y) d y \\
\underline{\underline{4.1}} \int_{D} R^{0}(x, y, k)\left(\Delta+E-V^{0}(y)\right) \varphi^{+}(y) d y \\
\underline{\underline{3.6}} \int_{D}\left(\Delta_{y}+E-V^{0}(y)\right) R^{0}(x, y, k) \varphi^{+}(y) d y \\
+\int_{\partial D}\left(R^{0}(x, y, k) \frac{\partial \varphi^{+}}{\partial \nu_{y}}(y)-\frac{\partial R^{0}}{\partial \nu_{y}}(x, y, k) \varphi(y)\right) d y \\
\int_{D} \delta_{x}(y) \varphi^{+}(y) d y+\int_{\partial D}\left(R^{0}(x, y, k) \frac{\partial \varphi^{+}}{\partial \nu_{y}}(y)-\frac{\partial R^{0}}{\partial \nu_{y}}(x, y, k) \varphi(y)\right) d y .
\end{gathered}
$$

Using formulas 4.12, 4.13, we obtain formula

$$
\varphi^{-}(x)=\psi^{0}(x, k)+\int_{\partial D}\left(R^{0}(x, y, k) \frac{\partial \varphi^{+}}{\partial \nu_{y}}(y)-\frac{\partial R^{0}}{\partial \nu_{y}}(x, y, k) \varphi(y)\right) d y
$$

where $x \in \mathbb{R}^{d} \backslash \bar{D}$.

It follows from formulas (2.17), 2.20, 2.22 and from the corresponding properties of single and double layer potentials that the following formulas are valid:

$$
\begin{gathered}
\int_{\partial D} R^{0}\left(x+0 \nu_{x}, y, k\right) \frac{\partial \varphi^{+}}{\partial \nu_{y}}(y) d y=\int_{\partial D} R^{0}\left(x-0 \nu_{x}, y, k\right) \frac{\partial \varphi^{+}}{\partial \nu_{y}}(y) d y \\
\int_{\partial D} \frac{\partial R^{0}}{\partial \nu_{y}}\left(x+0 \nu_{x}, y, k\right) \varphi(y) d y=-\varphi(x)+\int_{\partial D} \frac{\partial R^{0}}{\partial \nu_{y}}\left(x-0 \nu_{x}, y, k\right) \varphi(y) d y
\end{gathered}
$$

where $\nu_{x}$ is the unit exterior normal to $\partial D$ at $x \in \partial D$, and the argument $x+0 \nu_{x}$ (or $\left.x-0 \nu_{x}\right)$ means that we evaluate function at $x+\varepsilon \nu_{x}\left(\right.$ or $\left.x-\varepsilon \nu_{x}\right), \varepsilon>0$, and then pass $\varepsilon \rightarrow+0$. 
Besides, the following equality follows from (3.26), (4.13) and from definition of function $\varphi^{+}$:

$$
\begin{gathered}
\left(\Delta_{x}-V^{0}(x)+E\right)\left(\int_{\partial D} R^{0}(x, y, k) \frac{\partial \varphi^{+}}{\partial \nu_{y}}(y) d y\right. \\
\left.-\int_{\partial D} \frac{\partial R^{0}}{\partial \nu_{y}}(x, y, k) \varphi(y) d y\right)=0
\end{gathered}
$$

where $x \in D$.

Formulas (4.9), (4.16) imply that the function on the right hand side of equation (4.14) is annihilated by operator $\Delta-V^{0}+E$ in $D$ and formula (4.15) together with property $\left.\varphi^{-}\right|_{\partial D}=\varphi$ imply that this function has limit zero as $x$ approaches $\partial D$ from $D$. Since $E$ is not a Dirichlet eigenvalue for operator $L_{V^{0}} \equiv-\Delta+V^{0}$ in $D$ the following formula is valid:

$$
\psi^{0}(x, k)+\int_{\partial D}\left(R^{0}(x, y, k) \frac{\partial \varphi^{+}}{\partial \nu_{y}}(y)-\frac{\partial R^{0}}{\partial \nu_{y}}(x, y, k) \varphi(y)\right) d y=0,
$$

where $x \in D$.

Define

$$
\psi(x)= \begin{cases}\varphi^{-}(x), & x \in \mathbb{R}^{d} \backslash \bar{D} \\ \varphi(x), & x \in \partial D \\ \varphi^{+}(x), & x \in D\end{cases}
$$

It follows from formulas (4.13), 4.17) together with formula 4.12 that $\psi$ satisfies $(3.19)$ in $\mathbb{R}^{d}$.

Using (2.17), 2.20), (2.22), (3.19), we obtain that $\psi \in C^{1}\left(\mathbb{R}^{d}, M_{n}(\mathbb{C})\right)$.

From formulas (2.21), (2.22) it follows that functions $r^{0}(x, y, k), \partial_{x_{j}} r^{0}(x, y, k)$, $j=1, \ldots, d$, are uniformly bounded for $y \in D, x \notin D$, $\operatorname{dist}(x, \partial D)>1$. This fact together with the property that $\psi^{0}(x, k)=e^{i k x} \mu^{0}(x, k), \mu^{0} \in W^{1, \infty}\left(\mathbb{R}^{d}, M_{n}(\mathbb{C})\right)$ and with formula $(3.19)$ imply that $\psi(x, k)=e^{i k x} \mu(x, k)$ with $\mu \in W^{1, \infty}\left(\mathbb{R}^{d}, M_{n}(\mathbb{C})\right)$.

Thus, we have shown that if equation $(2.26)$ has a solution $\varphi \in C^{1, \beta}\left(\partial D, M_{n}(\mathbb{C})\right)$, then equation (3.19) has a solution $\psi(x, k)=e^{i k x} \mu(x, k)$ with $\mu \in W^{1, \infty}\left(\mathbb{R}^{d}, M_{n}(\mathbb{C})\right)$ and $\left.\psi\right|_{\partial D}=\varphi$. It follows from the latter property that different solutions to (2.26) give rise to different solutions to (3.19). More precisely, if $\psi^{\prime}, \psi^{\prime \prime}$ are two solutions to $(3.19)$, then $\left.\psi^{\prime}\right|_{\partial D}=\left.\psi^{\prime \prime}\right|_{\partial D}=\varphi$. It follows that $\psi^{\prime}, \psi^{\prime \prime}$ are two solutions to (4.1) and hence $\left.\psi^{\prime}\right|_{D}=\left.\psi^{\prime \prime}\right|_{D}$. Finally, it follows from (3.19) that $\psi^{\prime}=\psi^{\prime \prime}$.

2. Restricting a solution to equation (3.19) to a solution to equation (2.26). Let $\psi(x, k)=e^{i k x} \mu(x, k), \mu \in W^{1, \infty}\left(\mathbb{R}^{d}, M_{n}(\mathbb{C})\right)$, be a solution to 3.19). It follows from inclusion $W^{1, \infty}\left(\mathbb{R}^{d}, M_{n}(\mathbb{C})\right) \subset C\left(\mathbb{R}^{d}, M_{n}(\mathbb{C})\right)$ that $\varphi=\left.\psi\right|_{\partial D}$ is a continuous function on $\partial D$. Repeating the proof of Theorem 2.1] we can see that $\varphi$ is a solution to $(2.26)$. We are going to show that $\varphi \in C^{2}\left(\partial D, M_{n}(\mathbb{C})\right)$.

It follows from formula $(3.19)$ that $\psi \in C^{1}\left(\mathbb{R}^{d}, M_{n}(\mathbb{C})\right)$. Since $A_{1}, \ldots, A_{d}$, $V$ have compact supports in $D$, formula 3.19 together with formulas 2.20 , 
2.22 , 2.21) also imply that $\psi \in C^{2}\left(\mathbb{R}^{d} \backslash D, M_{n}(\mathbb{C})\right)$. Hence $\varphi$ belongs to $C^{2}\left(\partial D, M_{n}(\mathbb{C})\right)$.

Now if $\psi^{\prime}$ and $\psi^{\prime \prime}$ are two solutions to (3.19) such that $\left.\psi^{\prime}\right|_{\partial D} \neq\left.\psi^{\prime \prime}\right|_{\partial D}$, then it is clear that they give rise to different solutions to $(2.26)$. If, otherwise, $\left.\psi^{\prime}\right|_{\partial D}=\left.\psi^{\prime \prime}\right|_{\partial D}$ then $\psi^{\prime}=\psi^{\prime \prime}$ as was shown in the end of the preceding part of this subsection.

We have shown that solutions $\varphi$ to 2.26 of class $C^{1, \beta}\left(\partial D, M_{n}(\mathbb{C})\right)$ are in bijective correspondence with solutions $\psi$ to $(3.19)$ such that $\psi(x, k)=e^{i k x} \mu(x, k)$, $\mu \in W^{1, \infty}\left(\mathbb{R}^{d}, M_{n}(\mathbb{C})\right)$. This finishes the proof of Lemma 4.4 .

\section{Aknowledgements}

This article was prepared in the framework of research carried out under the supervision of Prof. R. G. Novikov.

\section{References}

[AN] A.D. Agaltsov, R. G. Novikov, Riemann-Hilbert problem approach for two-dimensional flow inverse scattering, J. Math. Phys. 55 (10), 103502, 2014

[Al] G. Alessandrini, Stable determination of conductivity by boundary measurements, Appl. Anal. 27, 1988, 153-172

[Ar] S. Arians, Geometric approach to inverse scattering for the Schrödinger equation with magnetic and electric potentials, J. Math. Phys. 38 (6), 1997, 2761-2773

[BBS] S.V. Baykov, V.A. Burov, S. N. Sergeev, Mode tomography of moving ocean, Proc. of the 3rd European Conference on Underwater Acoustics, $1996,845-850$.

[BSSR] V. A. Burov, S. N. Sergeev, A.S. Shurup, O.D. Rumyantseva, Application of functional-analytical Novikov algorithm for the purposes of ocean tomography, Proceedings of Meetings on Acoustics 17, 070065, 2012

[Buk] A.L. Bukhgeim, Recovering a potential from Cauchy data in the twodimensional case, J. Inverse Ill-Posed Probl. 16 (1), 2008, 19-33

[CK] D. Colton, R. Kress, Integral equation methods in scattering theory. John Wiley, New York, 1983

[ER1] G. Eskin, J. Ralston, Inverse scattering problem for the Schrödinger equation with magnetic potential at a fixed energy, Commun. Math. Phys. 173, 1995, 173-199 
[ER2] G.Eskin, J. Ralston, Inverse scattering problems for the Schrödinger operators with external Yang-Mills Potentials, Partial differential equations and their applications, (Toronto, ON, 1995), 91-106

[ER3] G. Eskin, J. Ralston, Inverse scattering problems for Schrödinger operators with magnetic and electric potentials, Inverse problems in wave propagation, IMA Vol. Math. Appl. 90, 1997, 147-166

[Es] G.Eskin, Global uniqueness in the inverse scattering problem for the Schrödinger operator with external Yang-Mills potentials, Comm. Math. Phys. 222 (3), 2001, 503-531

[Fa1] L.D. Faddeev, Growing solutions of the Schrödinger equation, Soviet Phys. Dokl. 10, 1966, 1033-1035

[Fa2] L.D. Faddeev, The inverse problem in the quantum theory of scattering. II, Journal of Soviet Mathematics 5 (3), 1976, 334-396

[FKSU] D. Dos Santos Ferreira, D. Kenig, J. Sjöstrand, G. Uhlmann, Derermining a magnetic Schrödinger operator from partial Cauchy data, Comm. Math. Phys. 271 (2), 2007, 467-488

[GT] D. Gilbarg, N. S. Trudinger, Elliptic Partial Differential Equations of Second Order. Classics in Mathematics, Springer, 2001

[HN1] G.M. Henkin, R.G. Novikov, The $\bar{\partial}$-equation in the multidimensional inverse scattering problem, Uspekhi Mat. Nauk, 42 (3), 1987, 93-152 (in Russian); English Transl.: Russ. Math. Surv. 42 (3), 1987, 109-180

[HN2] G. M. Henkin, R. G. Novikov, A multidimensional inverse problem in quantum and acoustic scattering, Inverse Problems 8, 1988, 103-121

[HN3] G. M. Henkin, R. G. Novikov. The Yang-Mills Fields, the Radon-Penrose Transform, and the Cauchy-Riemann Equations. Several Complex Variables V. Encyclopaedia of Mathematical Sciences 54, 1993, 109-193

[IY] O. Yu. Imanuvilov, M. Yamamoto, Inverse problem by Cauchy data on an arbitrary sub-boundary for systems of elliptic equations, Inverse Problems 28, (2012), 095015 (30pp)

[IN1] M. I. Isaev, R. G. Novikov, Reconstruction of a potential from the impedance boundary map, Eurasian Journal of Mathematical and Computer Applications 1 (1), 2013, 5-28

[IN2] M. I. Isaev, R. G. Novikov, Effectivized Hölder-logarithmic stability estimates for the Gel'fand inverse problem, Inverse Problems 30 (9), 095006, 2014

[KU] K. Krupchyk, G. Uhlmann, Uniqueness in an inverse boundary problem for a magnetic Schrödinger operator with a bounded magnetic potential, Comm. Math. Phys. 327 (3), 2014, 993-1009 
[KLU] K. Krupchyk, M. Lassas, G. Uhlmann, Inverse problems with partial data for a magnetic Schrödinger operator in an infinite slab or on a bounded domain, Comm. Math. Phys. 312 (1), 2012, 87-126

[NSU] G. Nakamura, Z. Q. Sun, G. Uhlmann, Global identifiability for an inverse problem for the Schrödinger equation in a magnetic field, Math. Ann. 303 (3), (1995), 377-388

[Ni] F. Nicoleau, A stationary approach to inverse scattering for Schrödinger operators with first order perturbation, Comm. Part. Diff. Eq. 22 (3-4), (1997), 527-553

[No1] R. G. Novikov, Multidimensional inverse spectral problem for the equation $-\Delta \psi+(v(x)-E u(x)) \psi=0$, Funkt. Anal. i Pril. 22 (4), 1988, 11-22 (in Russian); English Transl.: Funct. Anal. and Appl. 22, 1988, 263-272

[No2] R. G. Novikov, The inverse scattering problem on a fixed energy level for the two-dimensional Schrödinger operator, J. Funct. Anal. 103 (2), 1992, 409-469

[No3] R. G. Novikov, $\bar{\partial}$-method with nonzero background potential. Application to inverse scattering for the two-dimensional acoustic equation, Commun. Partial Diff. Eq. 21, 1996, 597-618

[No4] R. G. Novikov, Formulae and equations for finding scattering data from the Dirichlet-to-Neumann map with nonzero background potential, Inv. Problems 21 (1), 2005, 257-270

[No5] R. G. Novikov, An effectivization of the global reconstruction in the Gel'fandCalderón inverse problem in three dimensions, Imaging microstructures, Contemp. Math. 494, 2009, 161-184

[No6] R. G. Novikov, An iterative approach to non-overdetermined inverse scattering at fixed energy, Mat. Sb. 206 (1), 2015, 131-146

[NS1] R. G. Novikov, M. Santacesaria, Global uniqueness and reconstruction for the multi-channel Gel'fand-Calderón inverse problem in two dimensions, Bull. Sci. Math. 135 (5), 2011, 421-434

[NS2] R. G. Novikov, M. Santacesaria, Mohochromatic recontruction algorithms for two-dimensional multi-channel inverse problems, Int. Math. Res. Not. IMRN (6), 2013, 1205-1229

[Pa] A.Panchenko, An inverse problem for the magnetic Schrödinger equation and quasi-exponential solutions of nonsmooth partial differential equations, Inv. Problems 18 (5), 2002, 1421-1434

[PSU] L.Päivärinta, M.Salo, G. Uhlmann, Inverse scattering for the magnetic Schrödinger operator, J. Funct. Anal. 259 (7), 2010, 1771-1798 
[RW] D. Roussef, K. B. Winters, Two-dimensional vector flow inversion by diffraction tomography, Inverse Problems 10, 1994, 687-697

[RE] M. N. Rychagov, H. Ermert, Reconstruction of fluid motion in acoustic diffraction tomography, J. Acoust. Soc. Am. 99 (5), 1996, 3029-3035

[Sa] M. Santacesaria, A Hölder-logarithmic stability estimate for an inverse problem in two dimensions, Journal of Inverse and Ill-Posed Posed Problems, 2014, DOI:10.1515/jiip-2013-0055

[ST1] R. Schrader, M. Taylor, Small $\hbar$-asymptotics for quantum partition functions associated to particles in external Yang-Mills potentials, Comm. Math. Phys. 92 (4), 1984, 555-594

[ST2] R. Schrader, M. Taylor, Semiclassical asymptotics, gauge fields and quantum chaos, J. Funct. Anal. 83 (2), 1989, 258-316

[Sh] T. Shiota, An inverse problem for the wave equation with first order perturbation, Amer. J. Math. 107 (1), 1985, 241-251

[TU] M. Taylor, A. Uribe, Semiclassical spectra of gauge fields, J. Funct. Anal. 110 (1), 1992, 1-46.

[Xi] Xiaosheng Li, Inverse scattering problem for the Schrödinger operator with external Yang-Mills potentials in two dimensions at fixed energy, Comm. Part. Diff. Eq. 30, 2005, 451-482 